\title{
Synthesis of a Hexasaccharide Repeating Unit from Bacillus anthracis Vegetative Cell Walls
}

\author{
Matthias A. Oberli, ${ }^{\dagger}$ Pascal Bindschädler, ${ }^{\dagger}$ Daniel B. Werz, ${ }^{[a]}$ \\ and Peter H. Seeberger* \\ Laboratory for Organic Chemistry, \\ Swiss Federal Institute of Technology (ETH) Zürich, \\ ETH-Hönggerberg, HCI F 315, \\ Wolfgang-Pauli-Str. 10, \\ CH-8093 Zürich, Switzerland \\ E-mail: seeberger@org.chem.ethz.ch \\ ${ }^{\dagger}$ These authors contributed equally.

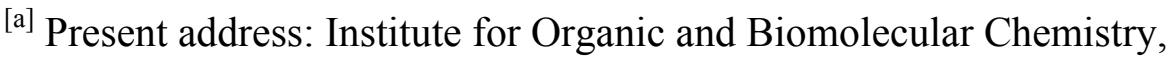 \\ University of Göttingen, Tammannstr. 2, \\ D-37077 Göttingen, Germany
}

\section{SUPPORTING INFORMATION - Part I Experimental Procedures}




\section{Table of Contents}

$\begin{array}{lr}\text { Full Reference } 8 & \text { S1-3 }\end{array}$

General Information $\quad$ S1-3

Experimental Procedure for compound $11 \quad$ S1-4

Experimental Procedure for compound $6 \quad$ S1-5

$\begin{array}{lr}\text { Experimental Procedure for compound } 13 & \text { S1-5 }\end{array}$

$\begin{array}{lr}\text { Experimental Procedure for compound } 14 & \text { S1-6 }\end{array}$

$\begin{array}{lr}\text { Experimental Procedure for compound } 7 & \text { S1-7 }\end{array}$

$\begin{array}{lr}\text { Experimental Procedure for compound } 9 & \text { S1-8 }\end{array}$

$\begin{array}{lr}\text { Experimental Procedure for compound } 16 & \text { S1-8 }\end{array}$

Experimental Procedure for compound $17 \quad$ S1-10

$\begin{array}{lr}\text { Experimental Procedure for compound } 18 & \text { S1-10 }\end{array}$

$\begin{array}{lr}\text { Experimental Procedure for compound } 19 & \text { S1-12 }\end{array}$

Experimental Procedure for compound $20 \quad$ S1-13

$\begin{array}{ll}\text { Experimental Procedure for compound } 22 & \text { S1-13 }\end{array}$

Experimental Procedure for compound $23 \quad$ S1-15

Experimental Procedure for compound $24 \quad$ S1-16

$\begin{array}{lr}\text { Experimental Procedure for compound } 25 & \text { S1-17 }\end{array}$

$\begin{array}{lr}\text { Experimental Procedure for compound } 26 & \text { S1-18 }\end{array}$

Experimental Procedure for compound $4 \quad$ S1-20

$\begin{array}{lr}\text { Experimental Procedure for compound 27 } & \text { S1-21 }\end{array}$

$\begin{array}{lr}\text { Experimental Procedure for compound } 1 & \text { S1-22 }\end{array}$

Assignment of Diagnostic NMR-Signals $\quad$ S1-23

Comparison of Hexasaccharide 1 to Isolated Polysaccharide $3 \quad$ S1-24

References in Supporting Information $\quad$ S1-26 
Full Reference 8. Verez-Bencomo, V.; Fernández-Santana, V.; Hardy, E.; Toledo, M. E.; Rodríguez, M. C.; Heynngnezz, L.; Rodriguez, A.; Baly, A.; Herrera, L.; Izquierdo, M.; Villar, A.; Valdés, Y.; Cosme, K.; Deler, M. L.; Montane, M.; Garcia, E.; Ramos, A.; Aguilar, A.; Medina, E.; Toraño, G.; Sosa, I.; Hernandez, I.; Martínez, R.; Muzachio, A.; Carmenates, A.; Costa, L.; Cardoso, F.; Campa, C.; Diaz, M.; Roy, R. Science 2004, 305, 522-525.

General Methods. All chemicals used were reagent grade and used as supplied except where noted. Dichloromethane $\left(\mathrm{CH}_{2} \mathrm{Cl}_{2}\right)$ and diethyl ether $\left(\mathrm{Et}_{2} \mathrm{O}\right)$ were purchased from JT Baker and purified by a Cycle-Tainer Solvent Delivery System. Pyridine, triethylamine and acetonitrile $\left(\mathrm{CH}_{3} \mathrm{CN}\right)$ were refluxed over calcium hydride and distilled. Triflic anhydride $\left(\mathrm{Tf}_{2} \mathrm{O}\right)$ was stirred over $\mathrm{P}_{2} \mathrm{O}_{5}$ and distilled. Boron trifluoride diethyletherate $\left(\mathrm{BF}_{3} \cdot \mathrm{OEt}_{2}\right)$ was diluted with diethyl ether and distilled from $\mathrm{Ca}(\mathrm{OH})_{2}$. Solvents for chromatography and workup procedures were distilled. Reactions were performed under an argon atmosphere except where noted. Analytical thin-layer chromatography was performed on E. Merck silica gel $60 \mathrm{~F}_{254}$ plates $(0.25 \mathrm{~mm})$. Compounds were visualized by UV-light at $254 \mathrm{~nm}$ and by dipping the plates in a cerium sulfate ammonium molybdate (CAM) solution or a sulfuric acid/methanol solution followed by heating. Liquid chromatography was performed using forced flow of the indicated solvent on Fluka silica gel 60 (230-400 mesh). ${ }^{1} \mathrm{H}$ NMR spectra were obtained on a Varian VXR300 (300 MHz), Bruker DRX500 (500 MHz), and Bruker AV600 (600 MHz) and are reported in parts per million $(\delta)$ relative to the resonance of the solvent or to TMS $(0.00 \mathrm{ppm})$. Coupling constants $(J)$ are reported in Hertz $(\mathrm{Hz}) .{ }^{13} \mathrm{C}$ NMR spectra were obtained on a Varian VXR-300 (75 MHz), Bruker DRX500 (125 MHz), and Bruker AV600 $(150 \mathrm{MHz})$ and are reported in $\delta$ relative to the resonance of the solvent or to TMS (0.00 ppm). IR Spectra: Measured as $1-2 \% \mathrm{CHCl}_{3}$ solution on a Perkin-Elmer-782 spectrophotometer or neat on a Perkin-Elmer-100 FT-IR spectrometer. Recycling preparative 
size exclusion HPLC (LC-9101, Japan Analytical Industry Co.); flow rate: $3.5 \mathrm{~mL} / \mathrm{min}$; solvent: $\mathrm{CHCl}_{3}$. Optical rotations $[\alpha]^{\mathrm{rt}}$ were measured on a Jasco DIP-370 polarimeter $(10 \mathrm{~cm}, 1 \mathrm{~mL}$ cell); the solvents and concentrations (in $\mathrm{g} / 100 \mathrm{~mL})$ are indicated. Highresolution mass spectra were performed by the MS service at the Laboratory for Organic Chemistry, ETH Zürich, and are given in $m / z$.

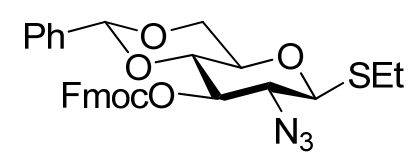

\section{Ethyl 2-Azido-4,6-O-benzylidene-2-deoxy-3-O-fluorenylmethoxycarbonyl-1-thio- $\beta$-D-}

glucopyranoside (11). A solution of ethyl 2-azido-4,6-O-benzylidene-2-deoxy-1-thio- $\beta$-Dglucopyranoside $10(2.0 \mathrm{~g}, 5.9 \mathrm{mmol})$ [1] in pyridine $(27 \mathrm{~mL})$ was treated at room temperature with $\mathrm{FmocCl}(3.1 \mathrm{~g}, 11.9 \mathrm{mmol})$. The yellow suspension was stirred at room temperature for $4 \mathrm{~h}$, concentrated and coevaporated once with toluene. The residue was taken up in EtOAc $(150 \mathrm{~mL})$, washed with $10 \%$ aqueous citric acid solution $(100 \mathrm{~mL})$, saturated aqueous $\mathrm{NaHCO}_{3}$ solution $(100 \mathrm{~mL})$, dried over $\mathrm{MgSO}_{4}$, filtered and concentrated. Flash column chromatography on silica gel $\left(1: 1 \rightarrow 0: 1\right.$, cyclohexane/ $\left.\mathrm{CH}_{2} \mathrm{Cl}_{2}\right)$ afforded $11(3.17 \mathrm{~g}$, 96\%) as a colorless foam. ${ }^{1} \mathrm{H} \mathrm{NMR}\left(\mathrm{CDCl}_{3}, 300 \mathrm{MHz}\right) \delta 1.37(\mathrm{t}, J=7.5 \mathrm{~Hz}, 3 \mathrm{H}), 2.73-2.88$ (m, 2H), $3.54(\mathrm{td}, J=9.7,4.9 \mathrm{~Hz}, 1 \mathrm{H}), 3.57(\mathrm{dd}, J=10.1,9.2 \mathrm{~Hz}, 1 \mathrm{H}), 3.73(\mathrm{t}, J=7.9 \mathrm{~Hz}$, 1H), 3.79 (t, $J=8.6 \mathrm{~Hz}, 1 \mathrm{H}), 4.30(\mathrm{t}, J=7.5 \mathrm{~Hz}, 1 \mathrm{H}), 4.37(\mathrm{dd}, J=10.6,5.0 \mathrm{~Hz}, 1 \mathrm{H}), 4.44-$ $4.55(\mathrm{~m}, 3 \mathrm{H}), 5.05(\mathrm{t}, J=9.6 \mathrm{~Hz}, 1 \mathrm{H}), 5.52(\mathrm{~s}, 1 \mathrm{H}), 7.21-7.47(\mathrm{~m}, 9 \mathrm{H}), 7.59-7.64(\mathrm{~m}, 2 \mathrm{H})$, 7.76-7.79 (m, 2H). ${ }^{13} \mathrm{C} \mathrm{NMR}\left(\mathrm{CDCl}_{3}, 75 \mathrm{MHz}\right) \delta 15.2,25.4,46.7,64.5,68.4,70.5,70.5,77.2$, $78.3,85.5,101.6,120.0,125.1,125.1,126.1,127.1,127.8,128.2,129.1,136.6,141.2,143.0$ 143.2, 154.2. $[\alpha]_{\mathrm{D}}^{\mathrm{rt}}=-75.0\left(c=1.0, \mathrm{CHCl}_{3}\right) . \mathrm{IR}\left(\mathrm{CHCl}_{3}\right): 3008,2879,2114,1757,1451$, 1386, 1314, 1100, $991 \mathrm{~cm}^{-1}$. MALDI-HRMS: $\mathrm{m} / \mathrm{z}$ calcd for $\mathrm{C}_{30} \mathrm{H}_{29} \mathrm{~N}_{3} \mathrm{O}_{6} \mathrm{SNa}[\mathrm{M}+\mathrm{Na}]^{+}$ 582.1669 , obsd 582.1679. 


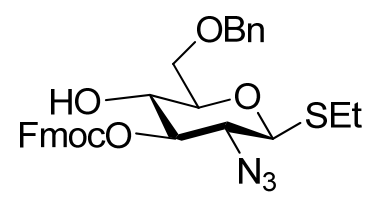

Ethyl

2-Azido-6-O-benzyl-2-deoxy-3-O-fluorenylmethoxycarbonyl-1-thio- $\beta$-D-

glucopyranoside (6). To a solution of $11(307 \mathrm{mg}, 0.55 \mathrm{mmol})$ and triethylsilane $(0.53 \mathrm{~mL}$, $3.3 \mathrm{mmol})$ in $\mathrm{CH}_{2} \mathrm{Cl}_{2}(5.5 \mathrm{~mL})$ at $0{ }^{\circ} \mathrm{C}$ was added dropwise trifluoroacetic acid $(0.25 \mathrm{~mL}, 3.3$ mmol). The solution was warmed to room temperature over $3.5 \mathrm{~h}$, quenched with saturated aqueous $\mathrm{NaHCO}_{3}$ solution, and diluted with $\mathrm{CH}_{2} \mathrm{Cl}_{2}$. The layers were separated and the aqueous phase was extracted once with $\mathrm{CH}_{2} \mathrm{Cl}_{2}$. The combined organic phases were dried over $\mathrm{MgSO}_{4}$, filtered and concentrated. Flash column chromatography on silica gel $(4: 1 \rightarrow$ 3:1, cyclohexane/EtOAc) afforded $6(255 \mathrm{mg}, 83 \%)$ as a colorless foam. ${ }^{1} \mathrm{H}$ NMR $\left(\mathrm{CDCl}_{3}\right.$, $300 \mathrm{MHz}) \delta 1.34(\mathrm{t}, J=7.3 \mathrm{~Hz}, 3 \mathrm{H}), 2.68-2.84(\mathrm{~m}, 2 \mathrm{H}), 3.00(\mathrm{~d}, J=3.4 \mathrm{~Hz}, 1 \mathrm{H}), 3.47-3.55$ (m, 2H), 3.72-3.83 (m, 3H), $4.31(\mathrm{t}, J=7.3 \mathrm{~Hz}, 1 \mathrm{H}), 4.40(\mathrm{~d}, J=10.0 \mathrm{~Hz}, 1 \mathrm{H}), 4.44-4.63(\mathrm{~m}$, $4 \mathrm{H}), 4.76(\mathrm{t}, J=9.5 \mathrm{~Hz}, 1 \mathrm{H}), 7.27-7.44(\mathrm{~m}, 9 \mathrm{H}), 7.61-7.66(\mathrm{~m}, 2 \mathrm{H}), 7.76-7.79(\mathrm{~m}, 2 \mathrm{H}) .{ }^{13} \mathrm{C}$ $\mathrm{NMR}\left(\mathrm{CDCl}_{3}, 75 \mathrm{MHz}\right) \delta 15.2,25.1,46.8,63.7,70.1,70.5,70.6,73.8,78.0,81.0,84.5$, $120.1,125.1,125.2,127.2,127.7,127.9,127.9,128.5,137.4,141.2,143.0,143.2,155.1$. $[\alpha]_{D}^{\mathrm{rt}}=-40.2\left(c=1.0, \mathrm{CHCl}_{3}\right) . \mathrm{IR}\left(\mathrm{CHCl}_{3}\right): 3498,3008,2872,2114,1753,1451,1387,1074$, $966 \mathrm{~cm}^{-1}$. MALDI-HRMS: $\mathrm{m} / \mathrm{z}$ calcd for $\mathrm{C}_{30} \mathrm{H}_{31} \mathrm{~N}_{3} \mathrm{O}_{6} \mathrm{SNa}[\mathrm{M}+\mathrm{Na}]^{+}$584.1826, obsd 584.1832.

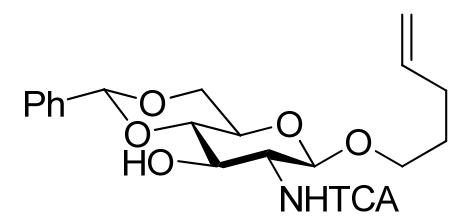

Pent-4-enyl 4,6-O-Benzylidene-2- $N$-trichloroacetyl- $\beta$-D-glucosaminopyranoside (13). To a solution of pent-4-enyl 2- $N$-trichloroacetyl- $\beta$-D-glucosaminopyranoside 12 (2.75 g, $7.0 \mathrm{mmol})$ [2] and benzaldehyde dimethylacetal $(2.15 \mathrm{~mL}, 21.0 \mathrm{mmol})$ in $\mathrm{CH}_{3} \mathrm{CN}(35 \mathrm{~mL})$ 
p-TsOH$\cdot \mathrm{H}_{2} \mathrm{O}(36 \mathrm{mg}, 0.2 \mathrm{mmol})$ was added. The mixture was stirred for $12 \mathrm{~h}$ at room temperature. The solution was diluted with EtOAc, quenched with saturated aqueous $\mathrm{NaHCO}_{3}$ solution and extracted. The aqueous phase was re-extracted with EtOAc. The combined organic extracts were washed with brine and dried over $\mathrm{MgSO}_{4}$, filtered and concentrated. Purification of the residue by flash column chromatography on silica gel $(6: 1 \rightarrow 2: 1$, cyclohexane/EtOAc) afforded $13(3.02 \mathrm{~g}, 90 \%)$ as a colorless solid. ${ }^{1} \mathrm{H}$ NMR $(300 \mathrm{MHz}$, $\left.\mathrm{CDCl}_{3}\right) \delta 1.65-1.74(\mathrm{~m}, 2 \mathrm{H}), 2.07-2.15(\mathrm{~m}, 2 \mathrm{H}), 2.85(\mathrm{~d}, J=3.1 \mathrm{~Hz}, 1 \mathrm{H}), 3.44-3.56(\mathrm{~m}, 4 \mathrm{H})$, $3.81(\mathrm{t}, J=10.1 \mathrm{~Hz}, 1 \mathrm{H}), 3.91(\mathrm{dt}, J=9.6,6.3 \mathrm{~Hz}, 1 \mathrm{H}), 4.34-4.44(\mathrm{~m}, 2 \mathrm{H}), 4.94(\mathrm{~d}, J=8.3$ Hz, 1H, H-1), 4.94-5.05 (m, 2H), 5.56 (s, 1H), 5.78 (ddt, J = 17.1, 10.2, $6.6 \mathrm{~Hz}, 1 \mathrm{H}), 6.93$ (d, $J=6.8 \mathrm{~Hz}, 1 \mathrm{H}), 7.35-7.40(\mathrm{~m}, 3 \mathrm{H}), 7.46-7.50(\mathrm{~m}, 2 \mathrm{H}) .{ }^{13} \mathrm{C} \mathrm{NMR}\left(75 \mathrm{MHz}, \mathrm{CDCl}_{3}\right) \delta 28.7$, $30.0,59.5,66.1,68.6,69.5,69.7,81.6,92.4,99.9,101.8,115.0,126.2,128.3,129.2,136.8$ 137.6, 162.1. IR $\left(\mathrm{CHCl}_{3}\right): 3432,3008,2880,1718,1520,1178,1096,997 \mathrm{~cm}^{-1} \cdot[\alpha]^{\mathrm{rt}} \mathrm{D}=-33.8$ $\left(c=1.0, \mathrm{CHCl}_{3}\right)$. MALDI-HRMS: $\mathrm{m} / \mathrm{z}$ calcd for $\mathrm{C}_{20} \mathrm{H}_{24} \mathrm{Cl}_{3} \mathrm{NO}_{6} \mathrm{Na}[\mathrm{M}+\mathrm{Na}]^{+}$502.0561, obsd 502.0578 .

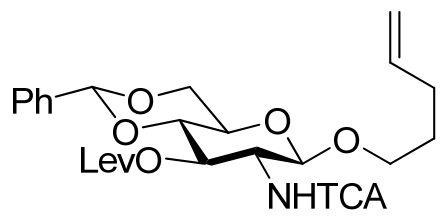

Pent-4-enyl 4,6-O-Benzylidene-3-O-levulinoyl-2- $N$-trichloroacetyl- $\beta$-D-glucosaminopyranoside (14). To a solution of $13(3.02 \mathrm{~g}, 6.3 \mathrm{mmol})$ in $\mathrm{CH}_{2} \mathrm{Cl}_{2}(65 \mathrm{~mL})$ at $0{ }^{\circ} \mathrm{C}$ DMAP (1.26 g, $10.3 \mathrm{mmol})$, DIPC (1.61 mL, $10.3 \mathrm{mmol})$ and levulinic acid (1.06 mL, $10.3 \mathrm{mmol})$ were added. The mixture was stirred for $3 \mathrm{~h}$ at room temperature, diluted with a mixture of cyclohexane/EtOAc $(2: 1,100 \mathrm{~mL})$, filtered over a plug of silica gel and concentrated. The crude product was purified by flash column chromatography on silica gel $(4: 1 \rightarrow 3: 1$, cyclohexane/EtOAc) to afforded $14(3.39 \mathrm{~g}, 93 \%)$ as a colorless foam. ${ }^{1} \mathrm{H}$ NMR $(300 \mathrm{MHz}$, $\left.\mathrm{CDCl}_{3}\right) \delta 1.61-1.71(\mathrm{~m}, 2 \mathrm{H}), 2.05-2.13(\mathrm{~m}, 2 \mathrm{H}), 2.13(\mathrm{~s}, 3 \mathrm{H}), 2.57-2.61(\mathrm{~m}, 2 \mathrm{H}), 2.70-2.75$ 
(m, 2H), $3.47(\mathrm{dt}, J=9.6,6.7 \mathrm{~Hz}, 1 \mathrm{H}), 3.51-3.59(\mathrm{~m}, 1 \mathrm{H}), 3.70-3.98(\mathrm{~m}, 4 \mathrm{H}), 4.32(\mathrm{dd}, J=$ 10.5, 4.9 Hz, 1H), 4.70 (d, $J=8.3 \mathrm{~Hz}, 1 \mathrm{H}, \mathrm{H}-1), 4.93-5.04$ (m, 2H), 5.45 (dd, $J=10.4,9.5$ $\mathrm{Hz}, 1 \mathrm{H}), 5.53(\mathrm{~s}, 1 \mathrm{H}), 5.78(\mathrm{ddt}, J=17.2,10.1,6.6 \mathrm{~Hz}, 1 \mathrm{H}), 6.95(\mathrm{~d}, J=8.7 \mathrm{~Hz}, 1 \mathrm{H})$, 7.35-7.38 (m, 3H), 7.44-7.48 (m, 2H). ${ }^{13} \mathrm{C} \mathrm{NMR}\left(75 \mathrm{MHz}, \mathrm{CDCl}_{3}\right) \delta 27.9,28.3,29.6,30.0$, $37.8,54.5,62.9,67.8,68.6,69.9,78.6,92.0,97.0,101.5,115.4,126.0,128.2,129.0,136.7$, 137.4, 161.9, 173.0, 205.7. IR $\left(\mathrm{CHCl}_{3}\right):$ 3008, 2936, 1720, 1515, 1374, 1248, 1128, 1088, 1052, $1002 \mathrm{~cm}^{-1} .[\alpha]_{\mathrm{D}}^{\mathrm{rt}}=+47.8\left(c=1.0, \mathrm{CHCl}_{3}\right)$. MALDI-HRMS: $\mathrm{m} / \mathrm{z}$ calcd for $\mathrm{C}_{25} \mathrm{H}_{30} \mathrm{Cl}_{3} \mathrm{NO}_{8} \mathrm{Na}[\mathrm{M}+\mathrm{Na}]^{+}$600.0929, obsd 600.0924 .

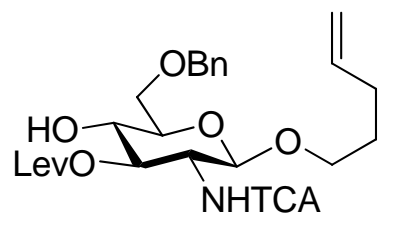

\section{Pent-4-enyl 6-O-Benzyl-3-O-levulinoyl-2- $N$-trichloroacetyl- $\beta$-D-glucosaminopyranoside}

(7). To an ice-cold mixture of $14(3.4 \mathrm{~g}, 5.9 \mathrm{mmol})$ and triethylsilane $(5.6 \mathrm{~mL}, 35.3 \mathrm{mmol})$ in $\mathrm{CH}_{2} \mathrm{Cl}_{2}(40 \mathrm{~mL})$ was slowly added trifluoroacetic anhydride $(0.84 \mathrm{~mL}, 5.9 \mathrm{mmol})$ and trifluoroacetic acid $(2.27 \mathrm{~mL}, 29.4 \mathrm{mmol})$. After stirring the mixture for $2.5 \mathrm{~h}$ at $0{ }^{\circ} \mathrm{C}$, the mixture was poured into saturated aqueous $\mathrm{NaHCO}_{3}$ solution and extracted twice with $\mathrm{CH}_{2} \mathrm{Cl}_{2}$. The combined organic extracts were washed with water, dried over $\mathrm{MgSO}_{4}$, filtered and concentrated. Purification of the residue by flash column chromatography on silica gel $\left(4: 1 \rightarrow 1: 1\right.$, cyclohexane/EtOAc) afforded $7(3.14 \mathrm{~g}, 92 \%)$ as a colorless oil. ${ }^{1} \mathrm{H}$ NMR $\left(300 \mathrm{MHz}, \mathrm{CDCl}_{3}\right) \delta 1.63-1.71(\mathrm{~m}, 2 \mathrm{H}), 2.05-2.14(\mathrm{~m}, 2 \mathrm{H}), 2.17(\mathrm{~s}, 3 \mathrm{H}), 2.47-2.65(\mathrm{~m}, 2 \mathrm{H})$, 2.77-2.83 (m, 2H), $3.28(\mathrm{~d}, J=2.0 \mathrm{~Hz}, 1 \mathrm{H}), 3.48(\mathrm{dt}, J=9.5,6.7 \mathrm{~Hz}, 1 \mathrm{H}), 3.58(\mathrm{dt}, J=9.7$, 4.4 Hz, 1H), 3.76-3.95 (m, 5H), 4.58 (d, J=8.3 Hz, 1H, H-1), 4.59 (d, J=12.0 Hz, 1H), 4.64 $(\mathrm{d}, J=12.0 \mathrm{~Hz}, 1 \mathrm{H}), 4.92-5.03(\mathrm{~m}, 2 \mathrm{H}), 5.14(\mathrm{dd}, J=10.9,8.9 \mathrm{~Hz}, 1 \mathrm{H}), 5.76$ (ddt, $J=17.1$, 10.1, 6.6 Hz, 1H), $6.70(\mathrm{~d}, J=8.8 \mathrm{~Hz}, 1 \mathrm{H}), 7.29-7.36(\mathrm{~m}, 5 \mathrm{H}) .{ }^{3} \mathrm{C} \mathrm{NMR}\left(75 \mathrm{MHz}, \mathrm{CDCl}_{3}\right) \delta$ $28.1,28.3,29.7,30.1,38.2,53.4,67.5,69.1,69.2,70.4,73.6,74.1,92.1,96.4,115.2,127.6$, 
127.7, 128.3, 128.9, 137.5, 137.7, 161.8, 173.4, 207.6. IR $\left(\mathrm{CHCl}_{3}\right): 3417,3008,2922,1718$, $1672,1514,1362,1156,1054 \mathrm{~cm}^{-1} \cdot[\alpha]_{D}^{\mathrm{rt}}=+50.2\left(c=1.0, \mathrm{CHCl}_{3}\right)$. MALDI-HRMS: $\mathrm{m} / \mathrm{z}$ calcd for $\mathrm{C}_{25} \mathrm{H}_{32} \mathrm{Cl}_{3} \mathrm{NO}_{8} \mathrm{Na}[\mathrm{M}+\mathrm{Na}]^{+}$602.1086, obsd 602.1096.

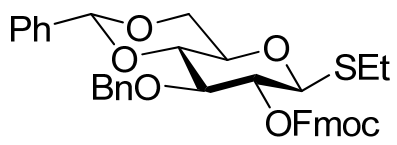

Ethyl 3-O-Benzyl-4,6-O-benzylidene-2-O-fluorenylmethoxycarbonyl-1-thio- $\beta$-Dglucopyranoside (9). A solution of ethyl 3-O-benzyl-4,6-O-benzylidene-1-thio- $\beta$-Dglucopyranoside $15(1.09 \mathrm{~g}, 2.71 \mathrm{mmol})$ [3] in pyridine $(12 \mathrm{~mL})$ was treated at room temperature with $\mathrm{FmocCl}(1.40 \mathrm{~g}, 5.42 \mathrm{mmol})$. The yellow suspension was stirred at room temperature for $2.5 \mathrm{~h}$, and concentrated. Flash column chromatography on silica gel $(1: 1 \rightarrow$ 0:1, cyclohexane/ $\left.\mathrm{CH}_{2} \mathrm{Cl}_{2}\right)$ afforded $9(1.53 \mathrm{~g}, 91 \%)$ as a colorless solid. ${ }^{1} \mathrm{H} \mathrm{NMR}\left(\mathrm{CDCl}_{3}, 300\right.$ MHz) $\delta 1.27(\mathrm{t}, J=7.5 \mathrm{~Hz}, 3 \mathrm{H}), 2.69-2.79(\mathrm{~m}, 2 \mathrm{H}), 3.51(\mathrm{td}, J=9.5,4.7 \mathrm{~Hz}, 1 \mathrm{H}), 3.75-3.89$ $(\mathrm{m}, 3 \mathrm{H}), 4.28(\mathrm{t}, J=7.2 \mathrm{~Hz}, 1 \mathrm{H}), 4.34-4.42(\mathrm{~m}, 2 \mathrm{H}), 4.55(\mathrm{dd}, J=10.3,7.2 \mathrm{~Hz}, 1 \mathrm{H}), 4.59(\mathrm{~d}$, $J=10.0 \mathrm{~Hz}, 1 \mathrm{H}), 4.73(\mathrm{~d}, J=11.8 \mathrm{~Hz}, 1 \mathrm{H}), 4.89(\mathrm{~d}, J=11.8 \mathrm{~Hz}, 1 \mathrm{H}), 4.90(\mathrm{dd}, J=10.3,8.4$ $\mathrm{Hz}, 1 \mathrm{H}), 5.59$ (s, 1H), 7.18-7.51 (m, 14H), 7.62-7.68 (m, 2H), 7.76-7.79 (m, 2H). ${ }^{13} \mathrm{C}$ NMR $\left(\mathrm{CDCl}_{3}, 75 \mathrm{MHz}\right) \delta 15.1,24.4,46.8,68.6,70.3,70.7,74.6,75.8,79.7,81.3,84.2,101.2$, $120.0,125.1,125.2,126.0,127.1,127.6,127.7,127.8,128.2,128.2,129.0,137.0,137.8$, 141.2, 143.1, 143.3, 154.4. $[\alpha]_{\mathrm{D}}^{\mathrm{rt}}=-48.9\left(c=1.0, \mathrm{CHCl}_{3}\right) . \mathrm{IR}\left(\mathrm{CHCl}_{3}\right): 3008,2876,1754$, 1451, 1385, 1321, 1097, 994, $972 \mathrm{~cm}^{-1}$. MALDI-HRMS: m/z calcd for $\mathrm{C}_{37} \mathrm{H}_{36} \mathrm{O}_{7} \mathrm{SNa}[\mathrm{M}+$ $\mathrm{Na}]^{+}$647.2074, obsd 647.2079.

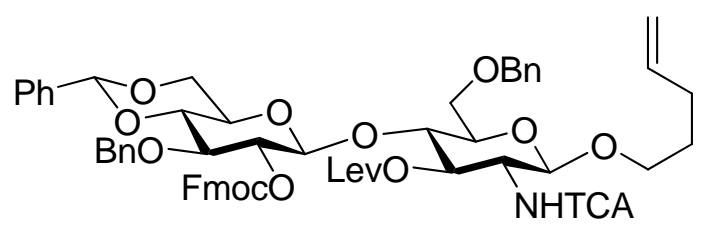




\section{glucopyranosyl-(1 $\rightarrow 4)$-6-O-benzyl-3-O-levulinoyl-2- $N$-trichloroacetyl- $\beta$-D-}

glucosaminopyranoside (16). Alcohol $7(580 \mathrm{mg}, 1.0 \mathrm{mmol})$ and thioglycoside $9(812 \mathrm{mg}$, $1.3 \mathrm{mmol}$ ) were coevaporated twice with toluene, dried in vacuo for $2 \mathrm{~h}$, and dissolved in $\mathrm{CH}_{2} \mathrm{Cl}_{2}(25 \mathrm{~mL})$. Tri-tert-butylpyrimidine (TTBP, $\left.1.2 \mathrm{~g}, 4.9 \mathrm{mmol}\right)$ and freshly activated $4 \AA$ molecular sieves were added, and the mixture was stirred at room temperature for $10 \mathrm{~min}$. At $0{ }^{\circ} \mathrm{C}$, MeOTf $(0.64 \mathrm{~mL}, 5.2 \mathrm{mmol})$ was added dropwise and the reaction was stirred at room temperature for $5 \mathrm{~h}$. Then, pyridine $(1.5 \mathrm{~mL})$ was added at $0{ }^{\circ} \mathrm{C}$, and the mixture was diluted with EtOAc, filtered over Celite, and washed with saturated aqueous $\mathrm{NaHCO}_{3}$ solution. The aqueous layer was re-extracted with EtOAc, and the combined organic phases were washed with brine, dried over $\mathrm{MgSO}_{4}$, filtered and concentrated. Flash column chromatography on silica $(9: 1 \rightarrow 4: 1$, cyclohexane/EtOAc) afforded $16(939 \mathrm{mg}, 82 \%)$ as a colorless foam. ${ }^{1} \mathrm{H}$ NMR $\left(\mathrm{CDCl}_{3}, 300 \mathrm{MHz}\right) \delta$ 1.63-1.74 (m, 2H), 2.05-2.17 (m, 2H), $2.17(\mathrm{~s}, 3 \mathrm{H}), 2.46-2.77$ (m, 4H), 3.09-3.20 (m, 2H), 3.38-3.57 (m, 4H), 3.63-3.97 (m, 5H), 4.18-4.33 (m, 4H), $4.54(\mathrm{~d}$, $J=8.2 \mathrm{~Hz}, 1 \mathrm{H}, \beta-\mathrm{H}), 4.55-4.63(\mathrm{~m}, 5 \mathrm{H}), 4.82(\mathrm{~d}, J=12.0 \mathrm{~Hz}, 1 \mathrm{H}), 4.96-5.06(\mathrm{~m}, 2 \mathrm{H}), 5.12$ $(\mathrm{dd}, J=10.6,9.0 \mathrm{~Hz}, 1 \mathrm{H}), 5.53(\mathrm{~s}, 1 \mathrm{H}), 5.79(\mathrm{ddt}, J=17.1,10.1,6.6 \mathrm{~Hz}, 1 \mathrm{H}), 6.71(\mathrm{~d}, J=8.8$ $\mathrm{Hz}, 1 \mathrm{H}), 7.16-7.47(\mathrm{~m}, 19 \mathrm{H}), 7.59-7.62(\mathrm{~m}, 2 \mathrm{H}), 7.77-7.81(\mathrm{~m}, 2 \mathrm{H}) .{ }^{13} \mathrm{C} \mathrm{NMR}\left(\mathrm{CDCl}_{3}\right.$, $75 \mathrm{MHz}) \delta 28.1,28.5,30.1,30.3,37.8,46.9,54.0,66.1,66.8,67.8,68.5,69.5,70.3,71.1$, $73.4,74.0,75.2,78.4,81.1,92.2,96.4,100.9,101.1,115.4,120.1,124.6,124.8,126.0,127.1$, $127.1,127.6,127.9,128.2,128.2,128.3,128.5,129.0,137.1,137.4,137.5,138.0,141.3$, $141.5,143.2,143.4,154.0,161.6,172.5,205.7 .[\alpha]^{\mathrm{rt}}=+41.0\left(c=1.0, \mathrm{CHCl}_{3}\right) . \mathrm{IR}\left(\mathrm{CHCl}_{3}\right)$ : $3417,3008,2876,1755,1719,1515,1452,1386,1364,1095,1004 \mathrm{~cm}^{-1}$. MALDI-HRMS: $\mathrm{m} / \mathrm{z}$ calcd for $\mathrm{C}_{60} \mathrm{H}_{62} \mathrm{Cl}_{3} \mathrm{NO}_{15} \mathrm{Na}[\mathrm{M}+\mathrm{Na}]^{+}$1164.3077, obsd 1164.3057. 


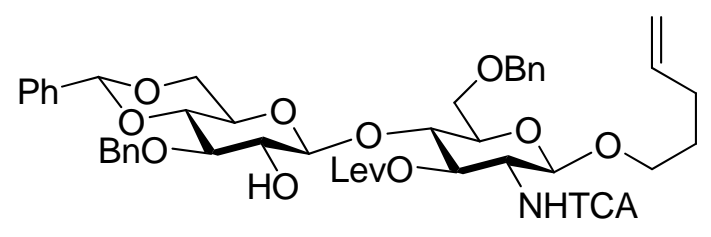

Pent-4-enyl 3-O-Benzyl-4,6-O-benzylidene- $\beta$-D-glucopyranosyl-(1 $\rightarrow 4)-6-O$-benzyl-3-Olevulinoyl-2- $N$-trichloroacetyl- $\beta$-D-glucosaminopyranoside (17). To a solution of 16 (930 mg, $0.81 \mathrm{mmol})$ in $\mathrm{CH}_{2} \mathrm{Cl}_{2}(32 \mathrm{~mL})$ triethylamine $(8 \mathrm{~mL})$ was added. The solution was stirred for $4 \mathrm{~h}$ at room temperature. The mixture was diluted with toluene $(50 \mathrm{~mL})$ and concentrated. The residue was coevaporated with toluene $(50 \mathrm{~mL})$. The crude product was purified by flash column chromatography on silica gel $(5: 1 \rightarrow 1: 1$, cyclohexane/EtOAc) to afford $17(691 \mathrm{mg}, 93 \%)$ as a colorless foam. ${ }^{1} \mathrm{H} \mathrm{NMR}\left(300 \mathrm{MHz}, \mathrm{CDCl}_{3}\right) \delta 1.63-1.73(\mathrm{~m}$, 2H), 2.09-2.16 (m, 2H), 2.16 (s, 3H), 2.48-2.80 (m, 4H), 3.27-3.39 (m, 2H), 3.47-3.80 (m, $6 \mathrm{H}), 3.86-4.00(\mathrm{~m}, 2 \mathrm{H}), 4.06-4.15(\mathrm{~m}, 2 \mathrm{H}), 4.28(\mathrm{~d}, J=7.5 \mathrm{~Hz}, 1 \mathrm{H}, \beta-\mathrm{H}), 4.34(\mathrm{dd}, J=10.3$, $4.9 \mathrm{~Hz}, 1 \mathrm{H}), 4.42(\mathrm{~d}, J=4.8 \mathrm{~Hz}, 1 \mathrm{H}), 4.58(\mathrm{~d}, J=8.3 \mathrm{~Hz}, 1 \mathrm{H}, \beta-\mathrm{H}), 4.60(\mathrm{~d}, J=12.8 \mathrm{~Hz}, 1 \mathrm{H})$, $4.69(\mathrm{~d}, J=11.5 \mathrm{~Hz}, 1 \mathrm{H}), 4.73(\mathrm{~d}, J=11.6 \mathrm{~Hz}, 1 \mathrm{H}), 4.90(\mathrm{~d}, J=11.8 \mathrm{~Hz}, 1 \mathrm{H}), 4.98-5.05(\mathrm{~m}$, 2H), $5.43(\mathrm{dd}, J=10.5,9.3 \mathrm{~Hz}, 1 \mathrm{H}), 5.53(\mathrm{~s}, 1 \mathrm{H}), 5.80(\mathrm{ddt}, J=17.1,10.2,6.6 \mathrm{~Hz}, 1 \mathrm{H})$, 7.21-7.33 (m, 10H), 7.37-7.42 (m, 3H), 7.44-7.48 (m, 2H), $7.63(\mathrm{~d}, J=9.6 \mathrm{~Hz}, 1 \mathrm{H})$. ${ }^{13} \mathrm{C}$ NMR $\left(75 \mathrm{MHz}, \mathrm{CDCl}_{3}\right) \delta 27.9,28.3,29.9,30.0,37.6,53.8,66.2,67.6,68.5,70.2,71.5$, $73.5,74.1,74.3,75.2,80.2,81.0,92.1,96.4,101.1,103.0,115.3,125.9,127.7,127.9,127.9$, $128.1,128.2,128.4,128.4,128.9,137.2,137.4,137.5,138.3,161.7,172.7,205.8$. IR $\left(\mathrm{CHCl}_{3}\right): 3418,3028,1719,1516,1365,1233,1070 \mathrm{~cm}^{-1} \cdot[\alpha]_{D}^{\mathrm{rt}}=+51.4\left(c=0.5, \mathrm{CHCl}_{3}\right)$. MALDI-HRMS: $m / z$ calcd for $\mathrm{C}_{45} \mathrm{H}_{52} \mathrm{Cl}_{3} \mathrm{NO}_{13} \mathrm{Na}[\mathrm{M}+\mathrm{Na}]^{+}$942.2396, obsd 942.2413.

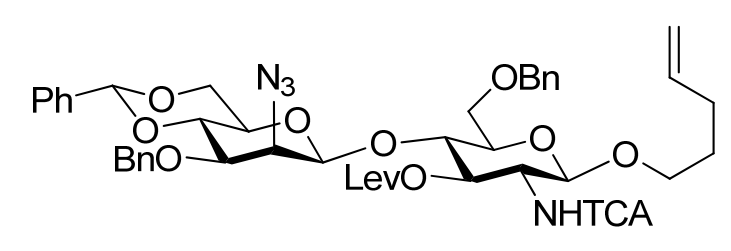


Pent-4-enyl 2-Azido-3-O-benzyl-4,6-di-O-benzylidene-2-deoxy- $\beta$-D-mannopyranosyl$(1 \rightarrow 4)-6-O$-benzyl-3-O-levulinoyl-2- $N$-trichloroacetyl- $\beta$-D-glucosaminopyranoside $\quad(18)$.

To a solution of $17(691 \mathrm{mg}, 0.75 \mathrm{mmol})$ in $\mathrm{CH}_{2} \mathrm{Cl}_{2}(10.5 \mathrm{~mL})$ pyridine $(1.4 \mathrm{~mL})$ and DMAP (one crystal) were added. The mixture was cooled to $0{ }^{\circ} \mathrm{C}$ and triflic anhydride $(0.7 \mathrm{~mL})$ was added. The reaction mixture was stirred for $3 \mathrm{~h}$ at $0{ }^{\circ} \mathrm{C}$, diluted with $\mathrm{CH}_{2} \mathrm{Cl}_{2}(50 \mathrm{~mL})$ and quenched with saturated aqueous $\mathrm{NaHCO}_{3}$ solution $(40 \mathrm{~mL})$. The phases were separated and the aqueous phase was extracted with $\mathrm{CH}_{2} \mathrm{Cl}_{2}(50 \mathrm{~mL})$. The combined organic extracts were washed with water $(100 \mathrm{~mL})$, brine $(100 \mathrm{~mL})$, dried over $\mathrm{MgSO}_{4}$, filtered and concentrated. The residue was purified by flash column chromatography on silica gel $(4: 1 \rightarrow 3: 1$, cyclohexane/EtOAc) to afford a colorless solid. This solid was dissolved in DMF (14 mL) and tetrabutylammonium azide $(1.2 \mathrm{~g}, 4.22 \mathrm{mmol})$ [4] was added. The solution was stirred for $14 \mathrm{~h}$ at $40-50{ }^{\circ} \mathrm{C}$. The mixture was diluted with water $(30 \mathrm{~mL})$ and extracted with a mixture of cyclohexane/EtOAc $(1: 1,3 \times 40 \mathrm{~mL})$. The combined organic phases were washed with water $(50 \mathrm{~mL})$, brine $(50 \mathrm{~mL})$, dried over $\mathrm{MgSO}_{4}$, filtered and concentrated. The crude product was purified by flash column chromatography on silica gel $(6: 1 \rightarrow 4: 1$, cyclohexane/EtOAc) to afford $18(622 \mathrm{mg}, 87 \%)$ as a colorless oil. ${ }^{1} \mathrm{H}$ NMR $\left(300 \mathrm{MHz}, \mathrm{CDCl}_{3}\right) \delta 1.64-1.72(\mathrm{~m}, 2 \mathrm{H})$, 2.06-2.14 (m, 2H), $2.17(\mathrm{~s}, 3 \mathrm{H}), 2.55-2.59(\mathrm{~m}, 2 \mathrm{H}), 2.71-2.75(\mathrm{~m}, 2 \mathrm{H}), 3.17(\mathrm{td}, J=9.6,4.8$ $\mathrm{Hz}, 1 \mathrm{H}), 3.49(\mathrm{dt}, J=9.5,6.7 \mathrm{~Hz}, 1 \mathrm{H}), 3.54(\mathrm{dd}, J=9.7,3.7 \mathrm{~Hz}, 1 \mathrm{H}), 3.61(\mathrm{dt}, J=9.6,2.7$ $\mathrm{Hz}, 1 \mathrm{H}), 3.73-3.82(\mathrm{~m}, 4 \mathrm{H}), 3.86-4.05(\mathrm{~m}, 4 \mathrm{H}), 4.30(\mathrm{dd}, J=10.6,4.8 \mathrm{~Hz}, 1 \mathrm{H}), 4.46(\mathrm{~d}, J=$ $12.0 \mathrm{~Hz}, 1 \mathrm{H}), 4.53\left(\mathrm{~d}, J=1.1 \mathrm{~Hz}, 1 \mathrm{H}, \mathrm{H}-1_{\beta-\mathrm{Man}}\right), 4.59$ (d, $\left.J=8.2 \mathrm{~Hz}, 1 \mathrm{H}, \mathrm{H}-1_{\mathrm{Glc}}\right), 4.66(\mathrm{~d}, J=$ $12.3 \mathrm{~Hz}, 1 \mathrm{H}), 4.71(\mathrm{~d}, J=12.0 \mathrm{~Hz}, 1 \mathrm{H}), 4.80(\mathrm{~d}, J=12.3 \mathrm{~Hz}, 1 \mathrm{H}), 4.93-5.04(\mathrm{~m}, 2 \mathrm{H}), 5.31$ (dd, $J=10.5,9.2 \mathrm{~Hz}, 1 \mathrm{H}), 5.56(\mathrm{~s}, 1 \mathrm{H}), 5.78(\mathrm{ddt}, J=17.1,10.2,6.6 \mathrm{~Hz}, 1 \mathrm{H}), 6.93(\mathrm{~d}, J=9.0$ $\mathrm{Hz}, 1 \mathrm{H}), 7.22-7.41(\mathrm{~m}, 13 \mathrm{H}), 7.46-7.50(\mathrm{~m}, 2 \mathrm{H}) .{ }^{13} \mathrm{C} \mathrm{NMR}\left(75 \mathrm{MHz}, \mathrm{CDCl}_{3}\right) \delta 28.0,28.6$, $29.8,29.9,37.8,55.9,63.2,67.1,68.2,69.0,72.0,72.7,73.6,74.2,75.2,76.4,78.2,92.3$, $99.6,100.6,101.5,114.9,125.9,127.4,127.8,127.9,128.1,128.2,128.4,128.5,128.9,137.1$, 137.5, 137.7, 137.8, 161.9, 172.5, 206.0. IR $\left(\mathrm{CHCl}_{3}\right):$ 3008, 2922, 2112, 1718, 1515, 1360, 
1265, 1156, 1090, $1048 \mathrm{~cm}^{-1} \cdot[\alpha]_{D}^{\mathrm{rt}}=+7.1\left(c=1.0, \mathrm{CHCl}_{3}\right)$. MALDI-HRMS: $\mathrm{m} / \mathrm{z}$ calcd for $\mathrm{C}_{45} \mathrm{H}_{51} \mathrm{Cl}_{3} \mathrm{~N}_{4} \mathrm{O}_{12} \mathrm{Na}[\mathrm{M}+\mathrm{Na}]^{+}$967.2461, obsd 967.2479.

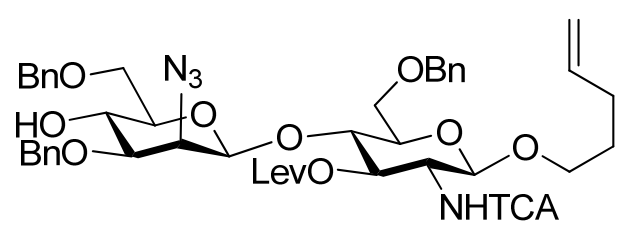

Pent-4-enyl 2-Azido-3,6-di-O-benzyl-2-deoxy- $\beta$-D-mannopyranosyl-(1 $\rightarrow$ 4)-6-O-benzyl-3O-levulinoyl-2- $N$-trichloroacetyl- $\beta$-D-glucosaminopyranoside (19). To a mixture of 18 (198 mg, $0.209 \mathrm{mmol})$ in $\mathrm{CH}_{2} \mathrm{Cl}_{2}(4 \mathrm{~mL})$ at $0{ }^{\circ} \mathrm{C}$ triethylsilane $(0.40 \mathrm{~mL}, 2.50 \mathrm{mmol})$ was added slowly, followed by boron trifluoride diethyletherate $(52 \mu \mathrm{L}, 0.418 \mathrm{mmol})$. After stirring the mixture for $2.5 \mathrm{~h}$ at $0{ }^{\circ} \mathrm{C}$, the mixture was poured into saturated aqueous $\mathrm{NaHCO}_{3}$ solution and extracted with $\mathrm{CHCl}_{3}(3 \times 30 \mathrm{~mL})$. The combined organic extracts were washed with water $(50 \mathrm{~mL})$, brine $(50 \mathrm{~mL})$, dried over $\mathrm{MgSO}_{4}$, filtered and concentrated. Purification of the residue by flash chromatography on silica gel $(5: 1 \rightarrow 2: 1$, cyclohexane/EtOAc) furnished acceptor $19(119 \mathrm{mg}, 60 \%)$ as a colorless foam. ${ }^{1} \mathrm{H}$ NMR $\left(300 \mathrm{MHz}, \mathrm{CDCl}_{3}\right) \delta$ 1.60-1.71 (m, 2H), 2.03-2.15 (m, 2H), $2.05(\mathrm{~s}, 3 \mathrm{H}), 2.46-2.65(\mathrm{~m}, 4 \mathrm{H}), 3.23-3.32(\mathrm{~m}, 2 \mathrm{H})$, $3.44(\mathrm{dt}, J=9.4,6.8 \mathrm{~Hz}, 1 \mathrm{H}), 3.62-4.06(\mathrm{~m}, 11 \mathrm{H}), 4.47-4.55(\mathrm{~m}, 5 \mathrm{H}), 4.57(\mathrm{~d}, J=12.1 \mathrm{~Hz}$, 1H), $4.64(\mathrm{~d}, J=11.7 \mathrm{~Hz}, 1 \mathrm{H}), 4.69(\mathrm{~d}, J=12.0 \mathrm{~Hz}, 1 \mathrm{H}), 4.93-5.02(\mathrm{~m}, 2 \mathrm{H}), 5.28(\mathrm{dd}, J=$ 10.4, 9.4 Hz, 1H), 5.77 (ddt, $J=17.1,10.2,6.6 \mathrm{~Hz}, 1 \mathrm{H}), 6.78(\mathrm{~d}, J=9.0 \mathrm{~Hz}, 1 \mathrm{H}), 7.23-7.37$ (m, 15H). ${ }^{13} \mathrm{C}$ NMR $\left(75 \mathrm{MHz}, \mathrm{CDCl}_{3}\right) \delta 27.9,28.7,29.7,30.0,37.9,56.1,60.9,67.8,68.5$, $69.1,70.1,71.5,71.9,73.4,73.7,74.4,74.6,80.5,92.3,98.6,100.8,114.8,127.5,127.7$, $127.8,128.0,128.4,128.4,128.5,137.3,137.6,137.8,161.8,172.9,206.1 . \mathrm{IR}\left(\mathrm{CHCl}_{3}\right): 3008$, $2879,2110,1720,1522,1364,1072 \mathrm{~cm}^{-1} ;[\alpha]^{\mathrm{rt}}=-56.3\left(c=1.0, \mathrm{CHCl}_{3}\right)$. MALDI-HRMS: $\mathrm{m} / \mathrm{z}$ calcd for $\mathrm{C}_{45} \mathrm{H}_{53} \mathrm{Cl}_{3} \mathrm{~N}_{4} \mathrm{O}_{12} \mathrm{Na}[\mathrm{M}+\mathrm{Na}]^{+}$969.2618, obsd 969.2609. 


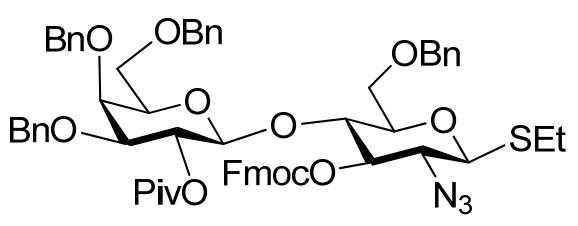

Ethyl $\quad(3,4,6$-Tri-O-benzyl-2-O-pivaloyl- $\beta$-D-galactopyranosyl)-(1 $\rightarrow 4)-2$-azido-6-Obenzyl-2-deoxy-3-O-fluorenylmethoxycarbonyl-1-thio- $\beta$-D-glucopyranoside (20). Acceptor 6 (514 mg, $0.915 \mathrm{mmol})$ and phosphate donor 8 (960 mg, $1.44 \mathrm{mmol})$ [5] were coevaporated with toluene $(3 \times)$, dried in vacuo for $2 \mathrm{~h}$, and dissolved in $\mathrm{CH}_{2} \mathrm{Cl}_{2}(20 \mathrm{~mL})$. At $-30{ }^{\circ} \mathrm{C}$, TMSOTf $(278 \mu \mathrm{L}, 1.44 \mathrm{mmol})$ was added dropwise. The reaction was warmed to $-20^{\circ} \mathrm{C}$ over $1 \mathrm{~h}$, quenched with pyridine $(2 \mathrm{~mL})$ and concentrated. The residue was coevaporated with toluene $(3 \times)$ and purified by flash column chromatography on silica $(9: 1$ $\rightarrow 7: 1$, cyclohexane/EtOAc) to afford $20(911 \mathrm{mg}, 92 \%)$ as a colorless foam. ${ }^{1} \mathrm{H}$ NMR $\left(\mathrm{CDCl}_{3}, 300 \mathrm{MHz}\right) \delta 1.14(\mathrm{~s}, 9 \mathrm{H}), 1.34(\mathrm{t}, J=7.3 \mathrm{~Hz}, 3 \mathrm{H}), 2.70-2.83(\mathrm{~m}, 2 \mathrm{H}), 3.38(\mathrm{dd}, J=$ $10.3,2.5 \mathrm{~Hz}, 1 \mathrm{H}), 3.43-3.49(\mathrm{~m}, 3 \mathrm{H}), 3.58-3.60(\mathrm{~m}, 2 \mathrm{H}), 3.74-3.75(\mathrm{~m}, 2 \mathrm{H}), 3.91-3.98(\mathrm{~m}$, $3 \mathrm{H}), 4.15(\mathrm{t}, J=7.3 \mathrm{~Hz}, 1 \mathrm{H}), 4.27(\mathrm{~d}, J=11.8 \mathrm{~Hz}, 1 \mathrm{H}), 4.32-4.54(\mathrm{~m}, 7 \mathrm{H}), 4.63(\mathrm{~d}, J=$ $11.8 \mathrm{~Hz}, 1 \mathrm{H}), 4.73(\mathrm{~d}, J=11.8 \mathrm{~Hz}, 1 \mathrm{H}), 4.79(\mathrm{~d}, J=11.5 \mathrm{~Hz}, 1 \mathrm{H}), 4.86(\mathrm{t}, J=9.5 \mathrm{~Hz}, 1 \mathrm{H})$, $5.30\left(\mathrm{dd}, J=10.1,7.9 \mathrm{~Hz}, 1 \mathrm{H}, \mathrm{H}-2_{\mathrm{Gal}}\right), 7.03-7.43(\mathrm{~m}, 25 \mathrm{H}), 7.51-7.53(\mathrm{~m}, 1 \mathrm{H}), 7.75-7.77(\mathrm{~m}$, 2H). ${ }^{13} \mathrm{C} \mathrm{NMR}\left(\mathrm{CDCl}_{3}, 75 \mathrm{MHz}\right) \delta 15.2,25.0,27.4,38.9,46.9,63.9,67.9,68.1,69.8,71.3$, $72.0,72.2,73.5,73.6,74.3,78.8,78.9,81.1,84.2,100.1,119.8,119.8,125.3,125.5,127.0$, $127.1,127.3,127.5,127.6,127.7,127.8,127.9,128.0,128.3,128.4,137.6,137.9,138.3$, $141.1,141.2,143.3,143.5,153.8,176.3 .[\alpha]^{\mathrm{rt}}=+7.0\left(c=1.0, \mathrm{CHCl}_{3}\right) . \mathrm{IR}\left(\mathrm{CHCl}_{3}\right): 3011$, $2872,2114,1754,1740,1600,1496,1478,1452,1365,1133,1090 \mathrm{~cm}^{-1}$. MALDI-HRMS: $m / z$ calcd for $\mathrm{C}_{62} \mathrm{H}_{67} \mathrm{~N}_{3} \mathrm{O}_{12} \mathrm{SNa}[\mathrm{M}+\mathrm{Na}]^{+} 1100.4338$, obsd 1100.4319 .

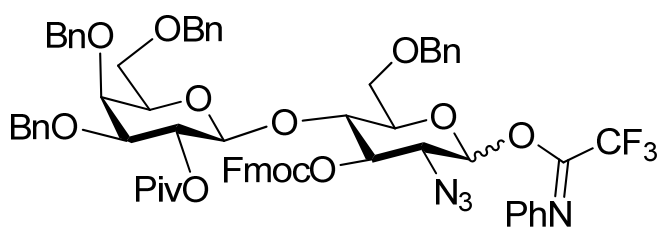




\section{3,4,6-Tri-O-benzyl-2-O-pivaloyl- $\beta$-D-galactopyranosyl-( $1 \rightarrow 4)-2$-azido-6-O-benzyl-2-}

\section{deoxy-3-O-fluorenylmethoxy-carbonyl- $\beta$-D-glucopyranosyl}

$N$-Phenyl

Trifluoroacetimidate (22). A solution of 20 (534 $\mathrm{mg}, 0.495 \mathrm{mmol})$ in a mixture of acetone $(16 \mathrm{~mL})$ and water $(4 \mathrm{~mL})$ was treated at room temperature with $N$-bromosuccinimide (308 mg, $1.73 \mathrm{mmol}$ ). After $40 \mathrm{~min}$, the solution was diluted with EtOAc and washed with $5 \%$ aqueous $\mathrm{Na}_{2} \mathrm{~S}_{2} \mathrm{O}_{3}$ solution. The aqueous layer was extracted twice with EtOAc, and the combined organic phases were washed with brine, dried over $\mathrm{MgSO}_{4}$, filtered and concentrated. Flash column chromatography $(7: 3 \rightarrow 3: 2$, cyclohexane/EtOAc) yielded the hemiacetal 21 (429 mg, 84\%) as a colorless foam. The obtained hemiacetal 21 (429 mg, $0.415 \mathrm{mmol})$ was dissolved in $\mathrm{CH}_{2} \mathrm{Cl}_{2}(10 \mathrm{~mL})$. At $0{ }^{\circ} \mathrm{C}, \mathrm{CF}_{3} \mathrm{C}(\mathrm{NPh}) \mathrm{Cl}(258 \mathrm{mg}, 1.25 \mathrm{mmol})$ [6] and $\mathrm{Cs}_{2} \mathrm{CO}_{3}(270 \mathrm{mg}, 0.83 \mathrm{mmol})$ were added and the resulting solution was stirred for $3 \mathrm{~h}$ at room temperature, diluted with $\mathrm{CH}_{2} \mathrm{Cl}_{2}$, filtered through Celite and concentrated. Flash column chromatography on silica $(9: 1 \rightarrow 5: 1$, cyclohexane/EtOAc) afforded 22 (423 mg, $85 \%)$ as a colorless oil. ${ }^{1} \mathrm{H} \mathrm{NMR}\left(\mathrm{CDCl}_{3}, 500 \mathrm{MHz}\right) \delta 1.05,1.08(2 \mathrm{~s}, 9 \mathrm{H}), 3.28(\mathrm{td}, J=9.5$, $2.3 \mathrm{~Hz}, 1 \mathrm{H}), 3.35-3.41(\mathrm{~m}, 1 \mathrm{H}), 3.51-4.10(\mathrm{~m}, 10 \mathrm{H}), 4.20-4.44(\mathrm{~m}, 7 \mathrm{H}), 4.47(\mathrm{dd}, J=10.7$, $7.1 \mathrm{~Hz}, 1 \mathrm{H}), 4.55,4.57(2 \mathrm{~d}, J=10.6,11.8 \mathrm{~Hz}, 1 \mathrm{H}), 4.67,4.68,4.71,4.74$ (4d, $J=12.0,12.1$, $11.7,11.5 \mathrm{~Hz}, 2 \mathrm{H}), 5.20,5.27\left(\mathrm{t}, \mathrm{dd}, J_{t}=9.4, J_{d d}=10.2,8.0 \mathrm{~Hz}, 1 \mathrm{H}\right), 6.48(\mathrm{br}, 1 \mathrm{H}), 6.75-6.78$ $(\mathrm{m}, 2 \mathrm{H}), 6.99-7.41(\mathrm{~m}, 28 \mathrm{H}), 7.44-7.46(\mathrm{~m}, 1 \mathrm{H}), 7.68-7.71(\mathrm{~m}, 2 \mathrm{H}) .{ }^{13} \mathrm{C} \mathrm{NMR}\left(\mathrm{CDCl}_{3}, 125\right.$ MHz) $\delta 27.5,27.5,29.9,38.9,39.0,47.0,47.1,60.9,63.3,67.2,67.4,68.4,68.4,70.0,70.1$, $71.5,72.2,72.2,72.4,72.5,72.9,73.0,73.3,73.7,73.8,74.0,74.5,75.1,75.6,81.2,81.3$, $100.2,100.3,119.5,120.0,120.1,120.1,124.8,125.6,125.7,127.3,127.3,127.6,127.6$, $127.8,127.9,127.9,127.9,128.0,128.2,128.2,128.3,128.3,128.6,128.6,128.8,128.8$, $129.0,129.4,137.9,138.0,138.0,138.3,138.3,138.6,138.6,141.4,141.5,141.5,143.2$, 143.3, 143.6, 143.6, 143.8, 143.9, 154.0, 176.5, 176.6. IR $\left(\mathrm{CHCl}_{3}\right): 3008,2871,2115,1759$, 1739, 1598, 1478, 1452, 1365, 1310, 1028, $909 \mathrm{~cm}^{-1}$. MALDI-HRMS: $\mathrm{m} / \mathrm{z}$ calcd for $\mathrm{C}_{68} \mathrm{H}_{67} \mathrm{~F}_{3} \mathrm{~N}_{4} \mathrm{O}_{13} \mathrm{Na}[\mathrm{M}+\mathrm{Na}]^{+}$1227.4549, obsd 1227.4558 . 


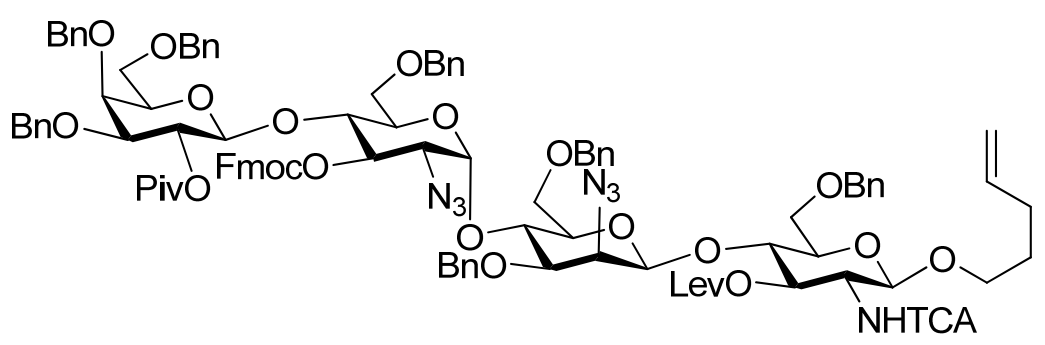

Pent-4-enyl 3,4,6-Tri-O-benzyl-2-O-pivaloyl- $\beta$-D-galactopyranosyl-(1 $\rightarrow$ 4)-2-azido-6-Obenzyl-2-deoxy-3-O-fluorenylmethoxycarbonyl- $\beta$-D-glucopyranosyl-(1 $\rightarrow 4)-2$-azido-3,6di-O-benzyl-2-deoxy- $\beta$-D-mannopyranosyl-(1 $\rightarrow 4)-6-O$-benzyl-3-O-levulinoyl-2- $N$ trichloroacetyl- $\beta$-D-glucosaminopyranoside (23). Disaccharide acceptor 19 (79 mg, $83 \mu \mathrm{mol})$ and disaccharide donor $22(151 \mathrm{mg}, 125 \mu \mathrm{mol})$ were coevaporated with toluene $(3 \times)$, dried in vacuo for $1 \mathrm{~h}$, and dissolved in a mixture of diethyl ether $(6 \mathrm{~mL})$ and $\mathrm{CH}_{2} \mathrm{Cl}_{2}$ (1.5 mL). At $-50{ }^{\circ} \mathrm{C}$, TMSOTf $(4.8 \mu \mathrm{L}, 25 \mu \mathrm{mol})$ was added, and the solution was warmed to $0{ }^{\circ} \mathrm{C}$ over $2.5 \mathrm{~h}$, quenched with pyridine $(0.1 \mathrm{~mL})$ and concentrated. The residue was coevaporated twice with toluene and purified by flash column chromatography on silica gel $\left(1: 0 \rightarrow 4: 1\right.$, cyclohexane/EtOAc) and recycling preparative $\mathrm{HPLC}\left(\mathrm{CHCl}_{3}\right)$ to afford $\mathbf{2 3}$ (131 mg, 80\%) as a colorless foam. ${ }^{1} \mathrm{H}$ NMR $\left(\mathrm{CDCl}_{3}, 500 \mathrm{MHz}\right) \delta 1.01(\mathrm{~s}, 9 \mathrm{H}), 1.57-1.65$ (m, 2H), $1.92(\mathrm{~s}, 3 \mathrm{H}), 2.01-2.08(\mathrm{~m}, 2 \mathrm{H}), 2.25-2.49(\mathrm{~m}, 4 \mathrm{H}), 3.08(\mathrm{dd}, J=10.9,7.7 \mathrm{~Hz}, 1 \mathrm{H}), 3.15-$ $3.20(\mathrm{~m}, 3 \mathrm{H}), 3.32-3.40(\mathrm{~m}, 2 \mathrm{H}), 3.49-3.55(\mathrm{~m}, 3 \mathrm{H}), 3.57-3.75(\mathrm{~m}, 7 \mathrm{H}), 3.79-3.93(\mathrm{~m}, 6 \mathrm{H})$, 4.00-4.08 (m, 4H), $4.12\left(\mathrm{~d}, J=8.0 \mathrm{~Hz}, 1 \mathrm{H}, \mathrm{H}-1_{\mathrm{Gal}}\right), 4.21(\mathrm{~d}, J=11.9 \mathrm{~Hz}, 1 \mathrm{H}), 4.28-4.56(\mathrm{~m}$, 13H), $4.62(\mathrm{~d}, J=11.9 \mathrm{~Hz}, 1 \mathrm{H}), 4.70(\mathrm{~d}, J=11.5 \mathrm{~Hz}, 1 \mathrm{H}), 4.88-4.96(\mathrm{~m}, 2 \mathrm{H}), 5.09(\mathrm{dd}, J=$ 10.8, 9.0 Hz, 1H), $5.21\left(\mathrm{dd}, J=10.2,7.9 \mathrm{~Hz}, 1 \mathrm{H}, \mathrm{H}-2_{\mathrm{Gal}}\right), 5.22(\mathrm{t}, J=10.0 \mathrm{~Hz}, 1 \mathrm{H}), 5.65(\mathrm{~d}, J$ $=3.8 \mathrm{~Hz}, 1 \mathrm{H}, \alpha-\mathrm{H}), 5.72(\mathrm{ddt}, J=17.0,10.3,6.6 \mathrm{~Hz}, 1 \mathrm{H}), 6.65(\mathrm{~d}, J=8.9 \mathrm{~Hz}, 1 \mathrm{H}), 6.95-7.41$

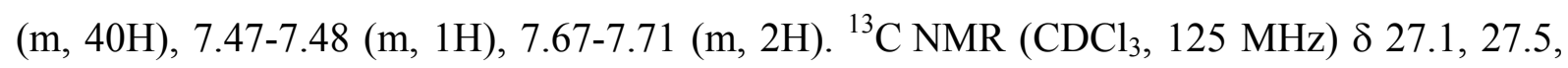
$27.9,28.9,29.8,30.2,37.8,38.9,47.1,56.5,60.9,61.2,67.3,68.4,68.9,69.3,69.4,69.9$, $70.8,71.0,71.3,71.4,71.7,72.1,72.5,73.5,73.6,73.7,73.8,73.8,74.0,74.1,74.4,74.8$, 75.0, 75.1, 77.1, 77.4, 81.3, 82.3, 92.6, 98.0, 98.1, 100.2, 101.1, 115.1, 120.0, 120.0, 125.6, 
$125.8,127.2,127.3,127.3,127.5,127.7,127.8,127.8,127.9,127.9,128.1,128.2,128.3$, $128.4,128.5,128.6,128.6,128.7,128.8,128.9,137.3,137.9,138.0,138.1,138.2,138.3$, $138.4,138.6,141.4,141.5,143.7,143.9,154.4,162.1,173.2,176.6,206.5 .[\alpha]^{\mathrm{rt}} \mathrm{D}=+15.4(c=$ 1.0, $\left.\mathrm{CHCl}_{3}\right)$. IR $\left(\mathrm{CHCl}_{3}\right): 3008,2870,2110,1738,1522,1497,1453,1363,1040 \mathrm{~cm}^{-1}$. MALDI-HRMS: $m / z$ calcd for $\mathrm{C}_{105} \mathrm{H}_{114} \mathrm{Cl}_{3} \mathrm{~N}_{7} \mathrm{O}_{24} \mathrm{Na}[\mathrm{M}+\mathrm{Na}]^{+}$1984.6873, obsd 1984.6851.

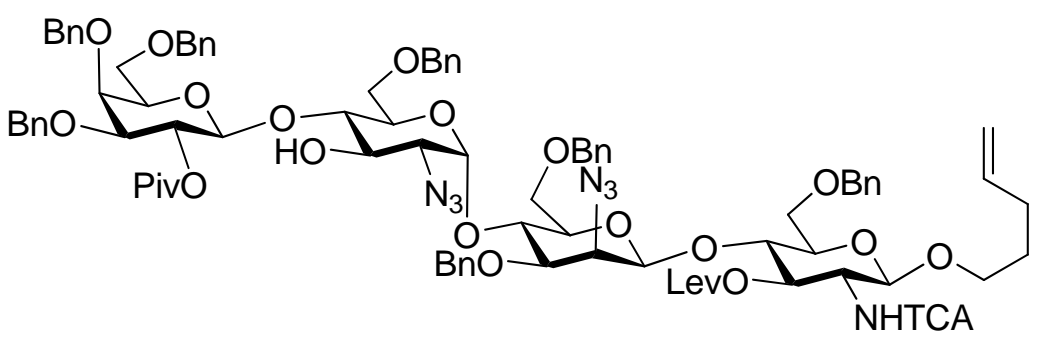

Pent-4-enyl $\quad 3,4,6$-Tri- $O$-benzyl-2-O-pivaloyl- $\beta$-D-galactopyranosyl-(1 $\rightarrow 4)$-2-azido-6-Obenzyl-2-deoxy- $\beta$-D-glucopyranosyl-( $1 \rightarrow 4)$-2-azido-3,6-di-O-benzyl-2-deoxy- $\beta$-Dmannopyranosyl-(1 $\rightarrow 4)$-6-O-benzyl-3-O-levulinoyl-2- $N$-trichloroacetyl- $\beta$-Dglucosaminopyranoside (24). To a solution of $23(131 \mathrm{mg}, 67 \mu \mathrm{mol})$ in $\mathrm{CH}_{2} \mathrm{Cl}_{2}(12 \mathrm{~mL})$ triethylamine $(3 \mathrm{~mL})$ was added. The solution was stirred for $17 \mathrm{~h}$ at room temperature. The mixture was diluted with toluene and concentrated. The residue was coevaporated twice with toluene. The crude product was purified by flash column chromatography on silica gel $(9: 1 \rightarrow$ 7:3, cyclohexane/EtOAc) to afford $24(85 \mathrm{mg}, 73 \%)$ as a colorless foam. ${ }^{1} \mathrm{H} \mathrm{NMR}\left(\mathrm{CDCl}_{3}\right.$, $500 \mathrm{MHz}) \delta 1.01(\mathrm{~s}, 9 \mathrm{H}), 1.56-1.64(\mathrm{~m}, 2 \mathrm{H}), 1.92(\mathrm{~s}, 3 \mathrm{H}), 1.99-2.06(\mathrm{~m}, 2 \mathrm{H}), 2.24-2.46(\mathrm{~m}$, 4H), $3.02(\mathrm{dd}, J=10.5,3.8 \mathrm{~Hz}, 1 \mathrm{H}), 3.18(\mathrm{dt}, J=9.7,3.0 \mathrm{~Hz}, 1 \mathrm{H}), 3.23(\mathrm{~d}, J=9.8 \mathrm{~Hz}, 1 \mathrm{H})$, $3.33(\mathrm{dd}, J=10.1,2.8 \mathrm{~Hz}, 1 \mathrm{H}), 3.35-3.43(\mathrm{~m}, 3 \mathrm{H}), 3.48-3.59(\mathrm{~m}, 9 \mathrm{H}), 3.67-4.02(\mathrm{~m}, 9 \mathrm{H})$, $4.14(\mathrm{~d}, J=12.2 \mathrm{~Hz}, 1 \mathrm{H}), 4.20-4.22(\mathrm{~m}, 2 \mathrm{H}), 4.30-4.51(\mathrm{~m}, 11 \mathrm{H}), 4.58(\mathrm{~d}, J=12.0 \mathrm{~Hz}, 1 \mathrm{H})$, $4.63(\mathrm{~d}, J=12.0 \mathrm{~Hz}, 1 \mathrm{H}), 4.81(\mathrm{~d}, J=11.6 \mathrm{~Hz}, 1 \mathrm{H}), 4.88-4.96(\mathrm{~m}, 2 \mathrm{H}), 5.20(\mathrm{dd}, J=10.4$, $9.5 \mathrm{~Hz}, 1 \mathrm{H}), 5.32(\mathrm{dd}, J=10.1,8.0 \mathrm{~Hz}, 1 \mathrm{H}), 5.55(\mathrm{~d}, J=3.8 \mathrm{~Hz}, 1 \mathrm{H}, \alpha-\mathrm{H}), 5.71$ (ddt, $J=$ 17.1, 10.2, 6.6 Hz, $1 \mathrm{H}), 6.62(\mathrm{~d}, J=8.9 \mathrm{~Hz}, 1 \mathrm{H}), 7.14-7.29(\mathrm{~m}, 35 \mathrm{H}) .{ }^{13} \mathrm{C} \mathrm{NMR}\left(\mathrm{CDCl}_{3}\right.$, 
$125 \mathrm{MHz}) \delta 27.5,27.9,28.9,29.7,30.2,37.8,39.0,56.5,61.0,62.8,67.7,68.7,68.8,69.3$, $69.4,69.5,70.4,70.7,71.1,71.4,71.8,72.4,72.5,73.2,73.8,73.9,73.9,74.0,74.2,74.2$, $74.7,74.8,75.0,79.2,81.2,82.4,92.6,97.9,98.1,101.0,101.2,115.1,127.4,127.5,127.6$, $127.7,127.7,127.8,127.9,128.0,128.0,128.1,128.2,128.2,128.3,128.4,128.5,128.5$, $128.6,128.7,128.7,128.8,128.8,128.9,137.3,137.7,137.7,137.9,138.2,138.2,138.4$, 138.5, 162.1, 173.3, 177.0, 206.5. $[\alpha]^{\mathrm{rt}}=+3.5\left(c=1.0, \mathrm{CHCl}_{3}\right)$. IR (neat): $3450,3352,3031$, 2909, 2870, 2108, 1718, 1529, 1454, 1365, 1275, 1136, 1049, $913 \mathrm{~cm}^{-1}$. MALDI-HRMS: $\mathrm{m} / \mathrm{z}$ calcd for $\mathrm{C}_{90} \mathrm{H}_{104} \mathrm{Cl}_{3} \mathrm{~N}_{7} \mathrm{O}_{22} \mathrm{Na}[\mathrm{M}+\mathrm{Na}]^{+}$1762.6192, obsd 1762.6211.

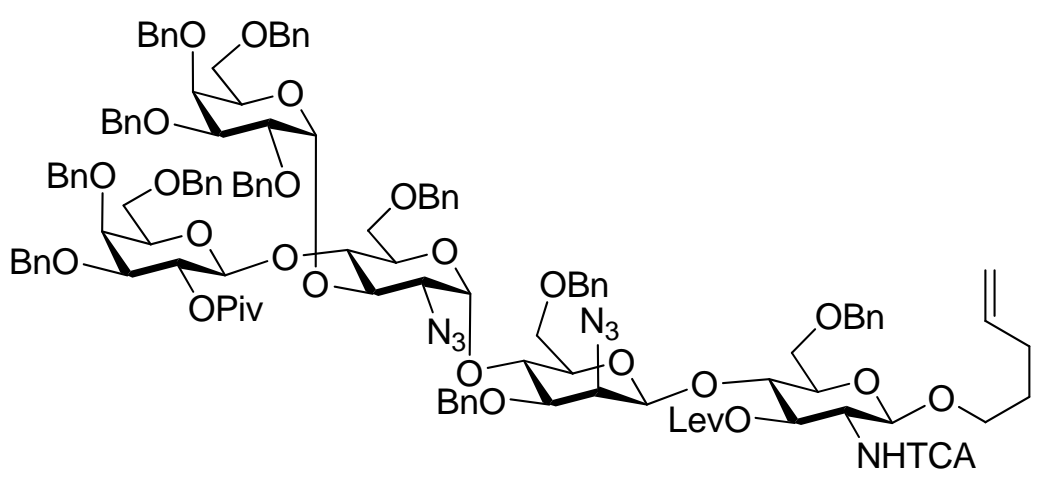

Pent-4-enyl $\quad 3,4,6$-Tri-O-benzyl-2-O-pivaloyl- $\beta$-D-galactopyranosyl-(1 $\rightarrow 4)$-[2,3,4,6-tetra$O$-benzyl- $\alpha$-D-galactopyranosyl-( $(\rightarrow 3)]$-2-azido-6-O-benzyl-2-deoxy- $\alpha$-D-glucopyranosyl$(1 \rightarrow 4)$-2-azido-3,6-di-O-benzyl-2-deoxy- $\beta$-D-mannopyranosyl-( $1 \rightarrow 4)-6-O$-benzyl-3-Olevulinoyl-2- $N$-trichloroacetyl- $\beta$-D-glucosaminopyranoside $\quad$ (25). $\quad$ Tetrasaccharide 24 (85 mg, $49 \mu \mathrm{mol})$ and galactosyl donor 5 (104 mg, $146 \mu \mathrm{mol})$ [7] were coevaporated with toluene $(3 \times)$ and dried in vacuo for $1 \mathrm{~h}$. The mixture was dissolved in diethyl ether/dichloromethane $(4: 1 ; 5 \mathrm{~mL})$ and cooled to $-45^{\circ} \mathrm{C}$. TMSOTf $(3 \mu \mathrm{L}, 15 \mu \mathrm{mol})$ was added, the mixture was stirred for $2 \mathrm{~h}$. During this time the mixture was allowed to warm to $0{ }^{\circ} \mathrm{C}$. The reaction was quenched with pyridine $(0.1 \mathrm{~mL})$ and concentrated. The residue was coevaporated twice with toluene and purified by column chromatography on silica gel $(9: 1 \rightarrow$ 3:1, cyclohexane/EtOAc) to afford pentasaccharide 25 (93 $\mathrm{mg}, 84 \%)$ as a colorless oil. 
${ }^{1} \mathrm{H}$ NMR $\left(\mathrm{CDCl}_{3}, 500 \mathrm{MHz}\right) \delta 1.04(\mathrm{~s}, 9 \mathrm{H}), 1.57-1.64(\mathrm{~m}, 2 \mathrm{H}), 1.90$ (s, 3H), 1.99-2.07 (m, 2H), 2.22-2.46 (m, 4H), $3.06(\mathrm{dd}, J=10.1,2.9 \mathrm{~Hz}, 1 \mathrm{H}), 3.09(\mathrm{dt}, J=9.7,3.0 \mathrm{~Hz}, 1 \mathrm{H}), 3.19-$ $3.22(\mathrm{~m}, 2 \mathrm{H}), 3.30-4.13(\mathrm{~m}, 28 \mathrm{H}), 4.27-4.47(\mathrm{~m}, 17 \mathrm{H}), 4.49(\mathrm{~d}, J=11.9 \mathrm{~Hz}, 1 \mathrm{H}), 4.50(\mathrm{~d}, J=$ $11.5 \mathrm{~Hz}, 1 \mathrm{H}), 4.56(\mathrm{~d}, J=11.7 \mathrm{~Hz}, 1 \mathrm{H}), 4.61(\mathrm{~d}, J=12.0 \mathrm{~Hz}, 1 \mathrm{H}), 4.62(\mathrm{~d}, J=12.4 \mathrm{~Hz}, 1 \mathrm{H})$, $4.67(\mathrm{~d}, J=11.6 \mathrm{~Hz}, 1 \mathrm{H}), 4.88-4.97(\mathrm{~m}, 2 \mathrm{H}), 5.21(\mathrm{t}, J=9.9 \mathrm{~Hz}, 1 \mathrm{H}), 5.24(\mathrm{dd}, J=10.1,8.1$ $\mathrm{Hz}, 1 \mathrm{H}), 5.58(\mathrm{~d}, J=4.2 \mathrm{~Hz}, 1 \mathrm{H}, \alpha-\mathrm{H}), 5.65(\mathrm{~d}, J=2.3 \mathrm{~Hz}, 1 \mathrm{H}, \alpha-\mathrm{H}), 5.72(\mathrm{ddt}, J=17.1$, 10.2, 6.6 Hz, 1H), $6.63(\mathrm{~d}, J=8.8 \mathrm{~Hz}, 1 \mathrm{H}), 7.03-7.29(\mathrm{~m}, 55 \mathrm{H}) .{ }^{13} \mathrm{C} \mathrm{NMR}\left(\mathrm{CDCl}_{3}, 125 \mathrm{MHz}\right)$ $\delta 27.5,27.9,28.9,29.7,30.2,37.8,38.9,56.5,61.0,62.9,68.2,68.4,68.8,69.4,69.5,69.5$, $70.3,70.7,71.2,71.4,71.6,71.7,72.1,72.6,72.8,73.3,73.3,73.6,73.7,73.8,74.0,74.3$, $74.6,74.7,74.8,74.8,75.4,75.7,76.4,78.5,81.5,82.1,92.6,96.6,97.6,98.2,100.1,101.1$, $115.2,127.1,127.2,127.2,127.3,127.5,127.6,127.6,127.7,127.8,127.9,127.9,128.0$, $128.0,128.1,128.2,128.2,128.2,128.3,128.3,128.3,128.4,128.4,128.5,128.5,128.6$, $128.6,128.7,128.8,128.9,137.4,137.9,138.1,138.1,138.2,138.2,138.6,138.7,138.7$, $139.2,139.4,139.5,162.1,173.2,176.3,206.4 .[\alpha]_{D}^{\mathrm{rt}}=+11.1\left(c=1.0, \mathrm{CHCl}_{3}\right) . \mathrm{IR}\left(\mathrm{CHCl}_{3}\right)$ : 3008, 2872, 2110, 1738, 1605, 1523, 1497, 1454, 1363, 1044, $913 \mathrm{~cm}^{-1}$. MALDI-HRMS: $\mathrm{m} / \mathrm{z}$ calcd for $\mathrm{C}_{124} \mathrm{H}_{138} \mathrm{Cl}_{3} \mathrm{~N}_{7} \mathrm{O}_{27} \mathrm{Na}[\mathrm{M}+\mathrm{Na}]^{+} 2284.8599$, obsd 2284.8544 .

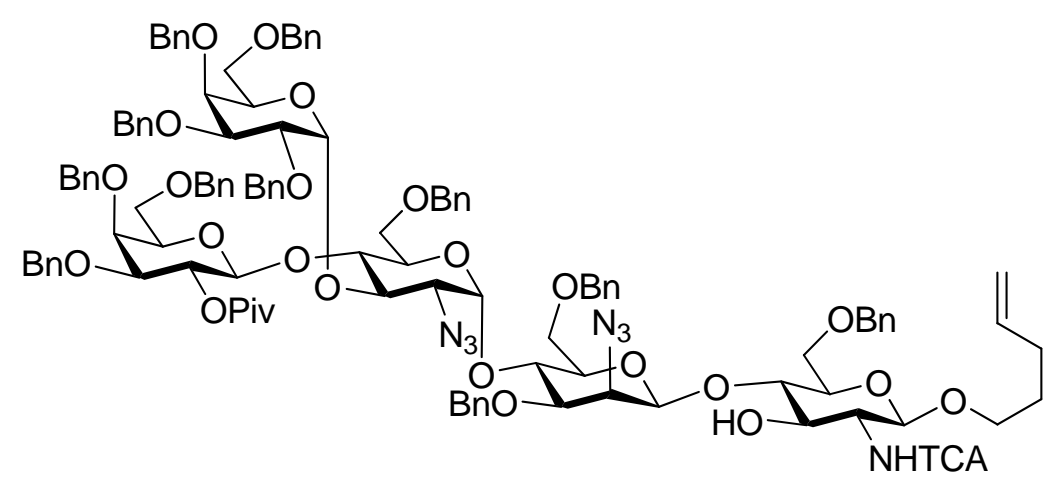

Pent-4-enyl $\quad 3,4,6$-Tri- $O$-benzyl-2-O-pivaloyl- $\beta$-D-galactopyranosyl-(1 $\rightarrow 4)$-[2,3,4,6-tetraO-benzyl- $\alpha$-D-galactopyranosyl-(1 $\rightarrow 3)]$-2-azido-6-O-benzyl-2-deoxy- $\alpha$-D-glucopyranosyl$(1 \rightarrow 4)-2$-azido-3,6-di-O-benzyl-2-deoxy- $\beta$-D-mannopyranosyl-( $1 \rightarrow 4)-6$-O-benzyl-2- $N$ - 
trichloroacetyl- $\beta$-D-glucosaminopyranoside (26). To a solution of $\mathbf{2 5}$ (26 mg, $11.5 \mu \mathrm{mol})$ in $\mathrm{CH}_{2} \mathrm{Cl}_{2}(2 \mathrm{~mL})$ pyridine $(42 \mu \mathrm{L})$, acetic acid $(28 \mu \mathrm{L})$ and hydrazine monohydrate $(0.8 \mu \mathrm{L}$, $23 \mu \mathrm{mol})$ were added. The solution was stirred for $5 \mathrm{~h}$ at room temperature. The reaction was quenched with acetone $(0.1 \mathrm{~mL})$ and concentrated. The residue was coevaporated twice with toluene and purified by flash column chromatography on silica gel $(1: 0 \rightarrow 3: 1$, cyclohexane/EtOAc) to afford $26(23.3 \mathrm{mg}, 94 \%)$ as a colorless foam. ${ }^{1} \mathrm{H} \mathrm{NMR}\left(\mathrm{CDCl}_{3}\right.$, $500 \mathrm{MHz}) \delta 1.05(\mathrm{~s}, 9 \mathrm{H}), 1.59-1.66(\mathrm{~m}, 2 \mathrm{H}), 2.03-2.09(\mathrm{~m}, 2 \mathrm{H}), 3.09(\mathrm{dd}, J=10.1,2.8 \mathrm{~Hz}$, $1 \mathrm{H}), 3.12-3.15(\mathrm{~m}, 1 \mathrm{H}), 3.20-3.23(\mathrm{~m}, 2 \mathrm{H}), 3.34-3.65(\mathrm{~m}, 17 \mathrm{H}), 3.76-4.15(\mathrm{~m}, 13 \mathrm{H}), 4.25-$ $4.50(\mathrm{~m}, 15 \mathrm{H}), 4.51(\mathrm{~d}, J=12.5 \mathrm{~Hz}, 1 \mathrm{H}), 4.57(\mathrm{~d}, J=11.7 \mathrm{~Hz}, 1 \mathrm{H}), 4.61(\mathrm{~d}, J=12.4 \mathrm{~Hz}, 1 \mathrm{H})$, $4.67(\mathrm{~d}, J=12.2 \mathrm{~Hz}, 1 \mathrm{H}), 4.69(\mathrm{~d}, J=11.1 \mathrm{~Hz}, 1 \mathrm{H}), 4.71(\mathrm{~d}, J=11.2 \mathrm{~Hz}, 1 \mathrm{H}), 4.76(\mathrm{~d}, J=$ $11.4 \mathrm{~Hz}, 1 \mathrm{H}), 4.82(\mathrm{~d}, J=8.4 \mathrm{~Hz}, 1 \mathrm{H}), 4.89-4.98(\mathrm{~m}, 2 \mathrm{H}), 5.25(\mathrm{dd}, J=10.0,8.1 \mathrm{~Hz}, 1 \mathrm{H})$, $5.45(\mathrm{~d}, J=4.1 \mathrm{~Hz}, 1 \mathrm{H}, \alpha-\mathrm{H}), 5.59(\mathrm{~d}, J=3.2 \mathrm{~Hz}, 1 \mathrm{H}, \alpha-\mathrm{H}), 5.73$ (ddt, $J=17.1,10.2,6.6 \mathrm{~Hz}$ $1 \mathrm{H}), 6.73(\mathrm{~d}, J=7.6 \mathrm{~Hz}, 1 \mathrm{H}), 7.04-7.31(\mathrm{~m}, 55 \mathrm{H}) .{ }^{13} \mathrm{C} \mathrm{NMR}\left(\mathrm{CDCl}_{3}, 125 \mathrm{MHz}\right) \delta 27.5,29.0$, $30.2,38.9,58.9,61.3,62.5,68.4,68.6,68.6,69.5,69.5,69.9,70.8,70.8,71.4,71.6,71.7$, 71.9, 72.1, 72.6, 72.7, 73.2, 73.3, 73.4, 73.7, 73.8, 73.8, 73.8, 74.1, 74.7, 74.7, 75.0, 75.0, $75.7,76.5,78.6,81.4,81.6,82.1,92.9,96.8,97.8,99.9,100.1,100.3,115.1,127.2,127.2$, $127.3,127.6,127.7,127.7,127.8,127.9,127.9,128.0,128.0,128.0,128.0,128.2,128.3$, $128.3,128.3,128.3,128.3,128.3,128.4,128.5,128.6,128.6,128.7,128.7,128.8,128.8$, $137.3,137.9,138.1,138.1,138.2,138.2,138.6,138.7,139.1,139.4,162.8,176.4 .[\alpha]_{D}^{\mathrm{rt}}=$ $+29.6\left(c=1.0, \mathrm{CHCl}_{3}\right) . \mathrm{IR}\left(\mathrm{CHCl}_{3}\right): 3438,3008,2870,2110,1734,1713,1522,1496,1454$, 1364, 1052, $910 \mathrm{~cm}^{-1}$. MALDI-HRMS: $\mathrm{m} / \mathrm{z}$ calcd for $\mathrm{C}_{119} \mathrm{H}_{132} \mathrm{Cl}_{3} \mathrm{~N}_{7} \mathrm{O}_{25} \mathrm{Na}[\mathrm{M}+\mathrm{Na}]^{+}$ 2186.8231 , obsd 2286.8234. 


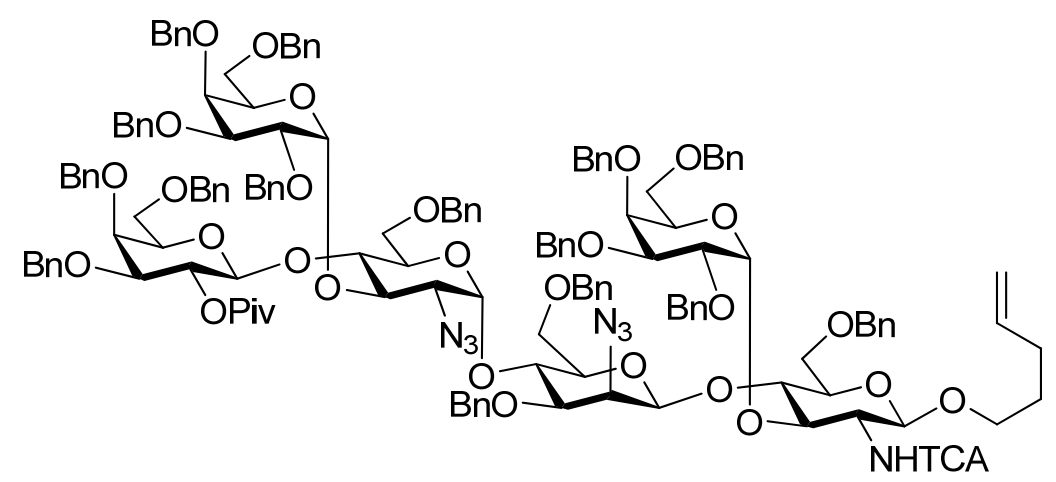

Pent-4-enyl $\quad 3,4,6$-Tri-O-benzyl-2-O-pivaloyl- $\beta$-D-galactopyranosyl-(1 $\rightarrow 4)$-[2,3,4,6-tetra$O$-benzyl- $\alpha$-D-galactopyranosyl-(1 $\rightarrow 3)]$-2-azido-6-O-benzyl-2-deoxy- $\alpha$-D-glucopyranosyl$(1 \rightarrow 4)$-2-azido-3,6-di-O-benzyl-2-deoxy- $\beta$-D-mannopyranosyl-( $1 \rightarrow 4)$-[2,3,4,6-tetra- $O$ benzyl- $\alpha$-D-galactopyranosyl-( $1 \rightarrow 3)]-6-O$-benzyl-2- $N$-trichloroacetyl- $\beta$-Dglucosaminopyranoside (4). Pentasaccharide 26 (74 mg, $34 \mu \mathrm{mol})$ and galactosyl donor 5 (73 mg, $103 \mu \mathrm{mol})$ [7] were coevaporated with toluene $(3 \times 10 \mathrm{~mL})$ and dried in vacuo for over night. The mixture was dissolved in diethyl ether/dichloromethane $(4: 1 ; 10 \mathrm{~mL})$ and cooled to $-50{ }^{\circ} \mathrm{C}$. TMSOTf $(1.0 \mu \mathrm{L}, 5.2 \mu \mathrm{mol})$ was added, the mixture was stirred for $4 \mathrm{~h}$. During this time the mixture was allowed to warm to $-3{ }^{\circ} \mathrm{C}$. The reaction was quenched with pyridine (few drops) and concentrated. The residue was purified by column chromatography on silica gel $(1: 0 \rightarrow 4: 1$, cyclohexane/EtOAc) to afford hexasaccharide $4(64.4 \mathrm{mg}, 70 \%)$ as a colorless oil. ${ }^{1} \mathrm{H}$ NMR (500 MHz, $\left.\mathrm{CDCl}_{3}\right) \delta 1.02(\mathrm{~s}, 9 \mathrm{H}), 1.49-1.60(\mathrm{~m}, 2 \mathrm{H}), 1.99-2.03$ (m, 2H), $2.96(\mathrm{dd}, J=9.8,3.7 \mathrm{~Hz}, 1 \mathrm{H}), 3.02(\mathrm{dd}, J=10.1,2.8 \mathrm{~Hz}, 1 \mathrm{H}), 3.12-3.18(\mathrm{~m}, 2 \mathrm{H})$, 3.27-3.34 (m, 2H), 3.36-3.44 (m, 4H), 3.47-3.52 (m, 5H), 3.53-3.56 (m, 2H), 3.70-3.79 (m, 4H), 3.83-3.86 (m, 4H), 3.88-3.95 (m, 8H), 4.03-4.12 (m, 6H), $4.16(\mathrm{~d}, J=10.9 \mathrm{~Hz}, 1 \mathrm{H})$, 4.22-4.42 (m, 11H), 4.44-4.52 (m, 9H), $4.55(\mathrm{~d}, J=11.8 \mathrm{~Hz}, 1 \mathrm{H}), 4.60-4.68(\mathrm{~m}, 5 \mathrm{H})$, 4.71-4.75 (m, 3H), 4.83-4.94 (m, 3H), $4.96(\mathrm{~d}, J=3.5 \mathrm{~Hz}, 1 \mathrm{H}, \alpha-\mathrm{H}), 5.22(\mathrm{dd}, J=10.0,8.1$ Hz, 1H), 5.59 (d, J = 4.2 Hz, 1H, $\alpha-\mathrm{H}), 5.64$ (br s, 1H, $\alpha-\mathrm{H}), 5.62-5.72(\mathrm{~m}, 1 \mathrm{H}), 7.04-7.27$ (m, $75 \mathrm{H}), 7.55(\mathrm{~d}, J=8.1 \mathrm{~Hz}, 1 \mathrm{H}) .{ }^{13} \mathrm{C} \mathrm{NMR}\left(125 \mathrm{MHz}, \mathrm{CDCl}_{3}\right) \delta 27.5,29.0,30.3,38.9$, $55.6,61.1,62.8,68.2,68.4,69.0,69.3,69.4,69.6,69.8,70.3,70.4,70.7,71.2,71.6,71.9$, 
$72.1,72.5,72.6,72.9,73.1,73.3,73.4,73.6,73.7,73.8,74.4,74.6,74.7,74.8,74.9,75.0$, $75.2,75.3,75.7,76.3,76.6,77.4,78.5,79.2,81.5,82.6,92.9,96.7,97.5,97.9,98.7,99.3$, $100.1,115.1,127.1,127.2,127.5,127.5,127.6,127.6,127.7,127.7,127.8,127.8,127.9$, $127.9,128.0,128.0,128.1,128.1,128.2,128.2,128.3,128.3,128.3,128.4,128.4,128.5$, $128.6,128.6,128.6,128.6,128.6,128.8,137.1,138.1,138.2,138.2,138.3,138.5,138.6$, 137.7, 138.8, 138.8, 138.9, 138.9, 139.2, 139.4, 139.5, 161.7, 176.3. IR $\left(\mathrm{CHCl}_{3}\right): 3008,2868$, $2109,1735,1711,1496,1454,1365,1098 \mathrm{~cm}^{-1} .[\alpha]_{D}^{\mathrm{rt}}=+37.2\left(c=0.5, \mathrm{CHCl}_{3}\right)$. MALDIHRMS: $\mathrm{m} / \mathrm{z}$ calcd for $\mathrm{C}_{153} \mathrm{H}_{166} \mathrm{Cl}_{3} \mathrm{~N}_{7} \mathrm{O}_{30} \mathrm{Na}[\mathrm{M}+\mathrm{Na}]^{+} 2709.0631$, obsd 2709.0610.

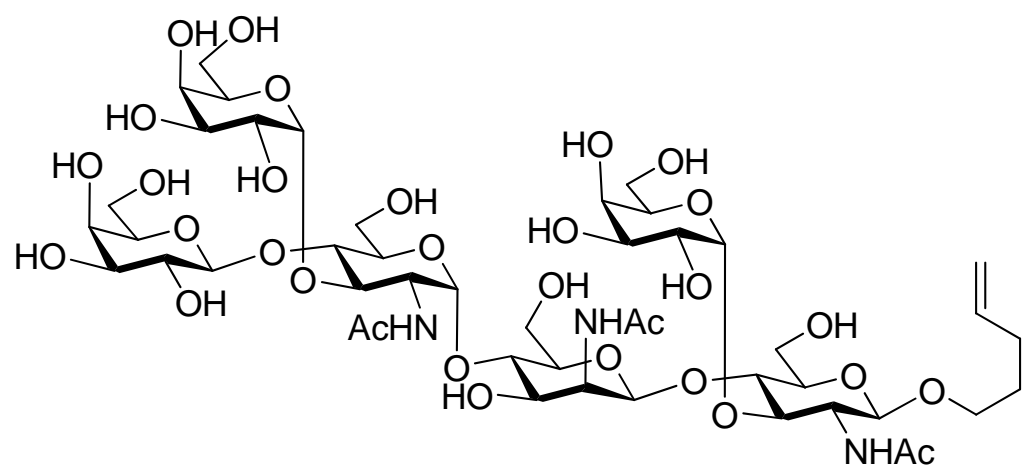

Pent-4-enyl $\beta$-D-Galactopyranosyl-( $1 \rightarrow 4)-[\alpha-D-$ galactopyranosyl- $(1 \rightarrow 3)]-\alpha-D-2-N$-acetylglucosaminopyranosyl-( $1 \rightarrow 4)-\beta-D-2-N$-acetyl-mannosaminopyranosyl-( $1 \rightarrow 4)-[\alpha-D-$ galactopyranosyl-(1 $\rightarrow 3)]-\beta-D-2-N$-acetyl-glucosaminopyranoside $(1)$. Hexasaccharide 4 (64 mg, $23.8 \mu \mathrm{mol})$ was dissolved in THF $(5 \mathrm{~mL})$ and ammonia was condensed into the reaction vessel at $-78{ }^{\circ} \mathrm{C}$ until the mixture reached a volume of about $25 \mathrm{~mL}$. Small pieces of carefully washed sodium were added to the reaction vessel affording a solution with a deep blue color. After 30 min the reaction was quenched by adding a few drops of methanol. Again, small pieces of sodium were added until the blue color persisted for at least $30 \mathrm{~min}$. The reaction was quenched by adding methanol $(5 \mathrm{~mL})$. The dry ice bath was removed, the reaction mixture was allowed to warm to room temperature in order to evaporate the ammonia. Acetic acid was added until an acidic $\mathrm{pH}$ was reached. The mixture was 
concentrated. The residue was dissolved in pyridine $(20 \mathrm{~mL})$. Acetic anhydride $(10 \mathrm{~mL})$ and DMAP (5 mg, $41 \mu \mathrm{mol}$ ) were added and the mixture was stirred over night. The mixture was diluted with toluene $(20 \mathrm{~mL})$ and concentrated. The crude product was purified by column chromatography on silica gel (toluene/acetone, 9:1 $\rightarrow$ 7:3) and recycling preparative HPLC $\left(\mathrm{CHCl}_{3}\right)$ affording $27(25 \mathrm{mg}, 57 \%)$ as a colorless solid. ${ }^{1} \mathrm{H} \mathrm{NMR}\left(500 \mathrm{MHz}, \mathrm{CDCl}_{3}\right) \delta$ 1.26-1.31 (m, 2H), 1.66-1.68 (m, 2H), $1.94(\mathrm{~s}, 3 \mathrm{H}), 1.95(\mathrm{~s}, 3 \mathrm{H}), 1.98(\mathrm{~s}, 3 \mathrm{H}), 1.99(\mathrm{~s}, 3 \mathrm{H})$, $2.00(\mathrm{~s}, 3 \mathrm{H}), 2.03(\mathrm{~s}, 3 \mathrm{H}), 2.05(\mathrm{~s}, 3 \mathrm{H}), 2.06(\mathrm{~s}, 3 \mathrm{H}), 2.09(\mathrm{~s}, 3 \mathrm{H}), 2.10(\mathrm{~s}, 6 \mathrm{H}), 2.10(\mathrm{~s}, 3 \mathrm{H})$, $2.11(\mathrm{~s}, 3 \mathrm{H}), 2.13(\mathrm{~s}, 3 \mathrm{H}), 2.14(\mathrm{~s}, 3 \mathrm{H}), 2.15(\mathrm{~s}, 3 \mathrm{H}), 2.15(\mathrm{~s}, 3 \mathrm{H}), 2.16(\mathrm{~s}, 3 \mathrm{H}), 2.18(\mathrm{~s}, 3 \mathrm{H})$, 3.39-3.42 (m, 1H), 3.45-3.50 (m, 2H), 3.60-3.65 (m, 1H), 3.66-3.69 (m, 1H), 3.74-3.79 (m, 2H), 3.82-3.89 (m, 4H), 4.00-4.04 (m, 1H), 4.12-4.16 (m, 5H), 4.20-4.32 (m, 3H), 4.37-4.53 (m, 6H), 4.78-4.80 (m, 1H), $4.91(\mathrm{~d}, J=6.7 \mathrm{~Hz}, 1 \mathrm{H}), 4.95-5.15(\mathrm{~m}, 10 \mathrm{H})$, $5.33-5.40(\mathrm{~m}, 3 \mathrm{H}), 5.42-5.45(\mathrm{~m}, 4 \mathrm{H}), 5.52(\mathrm{~d}, J=3.1 \mathrm{~Hz}, 1 \mathrm{H}), 5.75-5.83(\mathrm{~m}, 1 \mathrm{H}), 6.21(\mathrm{~d}, J$ $=8.6 \mathrm{~Hz}, 1 \mathrm{H}), 6.27-6.31(\mathrm{~m}, 2 \mathrm{H})$. MALDI-HRMS: $\mathrm{m} / \mathrm{z}$ calcd for $\mathrm{C}_{79} \mathrm{H}_{111} \mathrm{~N}_{3} \mathrm{O}_{47}[\mathrm{M}+\mathrm{Na}]^{+}$ 1876.6280, obsd 1876.6260. Compound 27 was dissolved in methanol ( $2 \mathrm{~mL})$ and sodium methoxide $(0.2 \mathrm{~mL}$ of a $0.5 \mathrm{M}$ solution in $\mathrm{MeOH})$ was added. The mixture was stirred over night. Amberlite (IR-120) acidic resin (washed and dried) was added until pH 7 was reached. The mixture was filtered, rinsed with $\mathrm{MeOH}$ and concentrated. The crude product was purified on Sep-Pack C18 Cartridges (1 g, water/methanol, 1:0 $\rightarrow$ 0:1). The product containing fractions were combined and lyophilized to afford $\mathbf{1}(13.5 \mathrm{mg}, 85 \%)$ as a colorless fluffy solid. ${ }^{1} \mathrm{H}$ NMR $\left(600 \mathrm{MHz}, \mathrm{D}_{2} \mathrm{O}\right) \delta 1.25-1.30(\mathrm{~m}, 2 \mathrm{H}), 1.63-165(\mathrm{~m}, 2 \mathrm{H}), 2.03(\mathrm{~s}, 6 \mathrm{H})$, $2.07(\mathrm{~s}, 3 \mathrm{H}), 3.50-4.11(\mathrm{~m}, 37 \mathrm{H}), 4.50\left(\mathrm{~d}, J=4.6 \mathrm{~Hz}, 1 \mathrm{H}, \mathrm{H}-2_{\mathrm{Man}}\right), 4.54$ (d, J= $7.8 \mathrm{~Hz}, 1 \mathrm{H}$, $\beta-\mathrm{H}), 4.54(\mathrm{~d}, J=8.3 \mathrm{~Hz}, 1 \mathrm{H}, \beta-\mathrm{H}), 4.90\left(\mathrm{~s}, 1 \mathrm{H}, \alpha-\mathrm{H}_{\mathrm{Man}}\right), 5.00-5.06(\mathrm{~m}, 2 \mathrm{H}), 5.26(\mathrm{~d}, J=3.6$ $\mathrm{Hz}, 1 \mathrm{H}, \alpha-\mathrm{H}), 5.49(\mathrm{~d}, J=3.9 \mathrm{~Hz}, 1 \mathrm{H}, \alpha-\mathrm{H}), 5.60(\mathrm{~d}, J=3.8 \mathrm{~Hz}, 1 \mathrm{H}, \alpha-\mathrm{H}), 5.84-5.91(\mathrm{~m}$, 1H). ${ }^{13} \mathrm{C}$ NMR $\left(150 \mathrm{MHz}, \mathrm{D}_{2} \mathrm{O}\right) \delta 22.7,24.8,24.9,24.9,30.5,32.0,55.5,56.4,57.3,62.1$, $62.6,62.9,63.3,63.4,63.7,71.3,71.3,71.5,71.6,71.7,72.0,72.2,72.5,73.6,73.6,73.8$, $74.7,75.2,75.9,77.2,77.7,77.8,77.9,78.2,78.4,79.4,100.2,100.9,101.1,101.7,103.4$ 
105.1, 117.5, 141.3, 176.8, 176.9, 178.3. ESI-HRMS: $m / z$ calcd for $\mathrm{C}_{47} \mathrm{H}_{79} \mathrm{~N}_{3} \mathrm{O}_{31} \mathrm{Na}[\mathrm{M}+$ $\mathrm{Na}^{+}$1204.4590, obsd 1204.4592.

\section{Assignment of Diagnostic NMR-Signals}

\section{Tetrasaccharide $\mathbf{2 3}$}

Analysis of ${ }^{1} \mathrm{H}-\mathrm{NMR}$ and HSQC spectra of tetrasaccharide 23 reveals the newly formed linkage to be alpha $\left({ }^{1} \mathrm{H}-\mathrm{NMR}: 5.65(\mathrm{~d}, J=3.8 \mathrm{~Hz}, \alpha-\mathrm{H})\right)$. The galactosyl $\mathrm{H}-1$ is, as expected, beta $\left({ }^{1} \mathrm{H}-\mathrm{NMR}: 4.12\left(\mathrm{~d}, J=8.0 \mathrm{~Hz}, \mathrm{H}-1_{\mathrm{Gal}}\right)\right.$, and has a cross peak in the COSY spectrum to the neighbouring H-2 ( ${ }^{1} \mathrm{H}-\mathrm{NMR}$ : 5.21 (dd, $\left.J=10.2,7.9 \mathrm{~Hz}, \mathrm{H}-2_{\mathrm{Gal}}\right)$. The other diagnostic anomeric protons were unambiguously assigned at earlier stages: The pentenyl moiety is $\beta$ linked (see ${ }^{1} \mathrm{H}-\mathrm{NMR}$ spectra of of 7 (4.58 (d, $\left.\left.J=8.3 \mathrm{~Hz}, \beta-\mathrm{H}_{\mathrm{Glc}}\right)\right), 13(4.94(\mathrm{~d}, J=8.3 \mathrm{~Hz}, \beta$ $\left.\left.\mathrm{H}_{\mathrm{Glc}}\right)\right), 14\left(4.70\left(\mathrm{~d}, J=8.3 \mathrm{~Hz}, \beta-\mathrm{H}_{\mathrm{Glc}}\right)\right), 16\left(4.54\left(\mathrm{~d}, J=8.2 \mathrm{~Hz}, \beta-\mathrm{H}_{\mathrm{Glc}}\right)\right), 17(4.58(\mathrm{~d}, J=$ $\left.\left.8.3 \mathrm{~Hz}, \beta-\mathrm{H}_{\mathrm{Glc}}\right)\right), \mathbf{1 8}\left(4.59\left(\mathrm{~d}, J=8.2 \mathrm{~Hz}, \beta-\mathrm{H}_{\mathrm{Glc}}\right)\right)$. The coupling of 9 and 7 involving the participating Fmoc group gave, as predicted, a $\beta$-linked product $\left({ }^{1} \mathrm{H}-\mathrm{NMR}\right.$ spectra of 17: 4.28 $(\mathrm{d}, J=7.5 \mathrm{~Hz}, \beta-\mathrm{H}))$. The inversion of glucose 17 at $\mathrm{C}-2$ yielded, as expected, selectively $\beta$-mannose azide $18\left({ }^{1} \mathrm{H}-\mathrm{NMR}: 4.53\left(\mathrm{~d}, J=1.1 \mathrm{~Hz}, \beta-\mathrm{H}_{\mathrm{Man}}\right)\right)$. Taken together, we believe to have consistently shown that the stereochemistry of tetrasaccharide $\mathbf{2 3}$ has been assigned correctly.

\section{Pentasaccharide 25}

The ${ }^{1} \mathrm{H}-\mathrm{NMR}$ spectrum of pentasaccharide $\mathbf{2 5}$ clearly shows two signals that are diagnostic for $\alpha$-linkages: $5.58(\mathrm{~d}, J=4.2 \mathrm{~Hz}, \alpha-\mathrm{H}), 5.65(\mathrm{~d}, J=2.3 \mathrm{~Hz}, \alpha-\mathrm{H})$. 


\section{Hexasaccharide 4}

${ }^{1}$ H-NMR, supported by HSQC analysis, clearly displays three signals that are diagnostic for $\alpha$-linkages: $4.96(\mathrm{~d}, J=3.5 \mathrm{~Hz}), 5.59(\mathrm{~d}, J=4.2 \mathrm{~Hz}), 5.64$ (br s). The coupling constants of the three remaining anomeric protons can not be determined since they are part of multiplets. However, their highfield chemical shifts $(\sim 4.70 \mathrm{ppm}, \sim 4.50 \mathrm{ppm}$ and $\sim 4.25 \mathrm{ppm})$ deduced from the HSQC spectra compare well with the corresponding signals of tetrasaccharide $\mathbf{2 3}$. As these linkages were installed and unambiguously assigned at an earlier stage, we believe to have consistently shown that also the stereochemistry of hexasaccharide $\mathbf{4}$ is correct.

\section{Hexasaccharide 1}

During the final deprotection using Birch conditions and subsequent $\mathrm{N}$ - and $\mathrm{O}$-acetylation, followed by $O$-deacetylation, the stereochemistry will not change. Additionally, on fully deprotected stage $\mathbf{1}$, the expected coupling signals of all diagnostic anomeric protons as well as the signal of $\mathrm{H}-2$ of the mannose residue are clearly visible: $4.50\left(\mathrm{~d}, J=4.6 \mathrm{~Hz}, \mathrm{H}-2_{\mathrm{Man}}\right.$ ), $4.54(\mathrm{~d}, J=7.8 \mathrm{~Hz}, \beta-\mathrm{H}), 4.54(\mathrm{~d}, J=8.3 \mathrm{~Hz}, \beta-\mathrm{H}), 4.90\left(\mathrm{~s}, \beta-\mathrm{H}_{\mathrm{Man}}\right), 5.26(\mathrm{~d}, J=3.6 \mathrm{~Hz}$, $\alpha-\mathrm{H}), 5.49(\mathrm{~d}, J=3.9 \mathrm{~Hz}, \alpha-\mathrm{H}), 5.60(\mathrm{~d}, J=3.8 \mathrm{~Hz}, \alpha-\mathrm{H})$. Therefore, we are sure that hexasaccharide $\mathbf{1}$ has the correct stereochemistry.

\section{Comparison of Hexasaccharide 1 to Isolated Polysaccharide 3}

The spectroscopic analysis of our hexameric structure (1) slightly differs from the one reported for isolated polysaccharide 3 [8], as expected. However, we believe that the two spectra match well enough to state that we confirmed the structure of repeating polysaccharide 3 by comparing it to synthesized hexasaccharide 1 . Comparison of diagnostic NMR-signals: 
Polysaccharide 3 [8]:

$\left({ }^{1} \mathrm{H}-\mathrm{NMR},{ }^{13} \mathrm{C}-\mathrm{NMR}\right): \alpha-G a l$ (5.64 (H-1), 98.8 (C-1)), $\alpha$-Gal (5.53 (H-1), 100.3(C-1)), $\alpha-$ GlcNAc (5.22 (H-1), 99.7 (C-1)), B-ManNAc (4.91 (H-1), 99.7 (C-1); 4.51 (H-2), 55.0 (C-2)), $\beta$-GlcNAc (4.67 (H-1), 101.9 (C-1)), $\beta$-Gal (4.44 (H-1), 104.1 (C-1)).

Hexasaccharide 1:

${ }^{1} \mathrm{H}-\mathrm{NMR}: 5.60(\mathrm{~d}, J=3.8 \mathrm{~Hz}, \alpha-\mathrm{H}), 5.49(\mathrm{~d}, J=3.9 \mathrm{~Hz}, \alpha-\mathrm{H}), 5.26(\mathrm{~d}, J=3.6 \mathrm{~Hz}, \alpha-\mathrm{H}), 4.90$ (s, $\left.\beta-\mathrm{H}_{\mathrm{Man}}\right), 4.54(\mathrm{~d}, J=8.3 \mathrm{~Hz}, \beta-\mathrm{H}), 4.54(\mathrm{~d}, J=7.8 \mathrm{~Hz}, \beta-\mathrm{H}), 4.50\left(\mathrm{~d}, J=4.6 \mathrm{~Hz}, \mathrm{H}-2_{\mathrm{Man}}\right)$. ${ }^{13} \mathrm{C}-\mathrm{NMR}: 105.1,103.4,101.7,101.1,100.9,100.2(6 \times \mathrm{C}-1)$. 


\section{References in Supporting Information}

[1] Garegg, P. J.; Konradsson, P.; Oscarson, S.; Ruda, K. Tetrahedron 1997, 53, $17727-$ 17734.

[2] Melean, L. G.; Love, K. R.; Seeberger, P. H. Carbohydr. Res. 2002, 337, 1893-1916.

[3] Veselý, J.; Rohlenová, A.; Džoganová, M.; Trnka, T.; Tišlerová, I.; Šaman, D.; Ledvina, M. Synthesis 2006, 699-705.

[4] Moss, R. A.; Terpinski, J.; Cox, D. P.; Denney, D. Z.; Krogh-Jespersen, K. J. Am Chem. Soc. 1985, 107, 2743-2748.

[5] Plante, O. J.; Andrade, R. B.; Seeberger, P. H. Org. Lett. 1999, 1, 211-214.

[6] Tamura, K.; Mizukami, H.; Maeda, K.; Watanabe, H.; Uneyama, K. J. Org. Chem. 1993, $58,32-35$.

[7] Tanaka, H.; Iwata, Y.; Takahashi, D.; Adachi, M.; Takahashi, T. J. Am. Chem. Soc. 2005, $127,1630-1631$.

[8] Choudhury, B.; Leoff, C.; Saile, E.; Wilkins, P.; Quinn, C. P.; Kannenberg, E. L.; Carlson, R. W. J. Biol. Chem. 2006, 281, 27932-27941. 